\title{
Organization of the Olfactory Pathway and Odor Processing in the Antennal Lobe of the Ant Camponotus floridanus
}

\author{
CHRISTINA ZUBE, ${ }^{1}$ CHRISTOPH JOHANNES KLEINEIDAM, ${ }^{1}$ \\ SEBASTIAN KIRSCHNER, ${ }^{2}$ JAKOB NEEF, ${ }^{1}$ AND WOLFGANG RÖSSLER ${ }^{1 *}$ \\ ${ }^{1}$ Department of Behavioral Physiology and Sociobiology, Biozentrum, University of \\ Würzburg, Würzburg, Germany \\ ${ }^{2}$ Department of Developmental and Comparative Psychology, Max Planck Institute for \\ Evolutionary Anthropology, Leipzig, Germany
}

\begin{abstract}
Ants rely heavily on olfaction for communication and orientation. Here we provide the first detailed structure-function analyses within an ant's central olfactory system asking whether in the carpenter ant, Camponotus floridanus, the olfactory pathway exhibits adaptations to processing many pheromonal and general odors. Using fluorescent tracing, confocal microscopy, and 3D-analyses we demonstrate that the antennal lobe (AL) contains up to $\approx 460$ olfactory glomeruli organized in seven distinct clusters innervated via seven antennal sensory tracts. The AL is divided into two hemispheres regarding innervation of glomeruli by either projection neurons (PNs) with axons leaving via the medial (m) or lateral (l) antennocerebral tract (ACT). M- and l-ACT PNs differ in their target areas in the mushroom-body calyx and lateral horn. Three additional ACTs project to the lateral protocerebrum only. We analyzed odor processing in AL glomeruli by retrograde loading of PNs with Fura-2 dextran and fluorimetric calcium imaging. Odor responses were reproducible and comparable across individuals. Calcium responses to pheromonal and nonpheromonal odors were very sensitive $\left(10^{-11}\right.$ dilution) and patterns were partly overlapping, indicating that processing of both odor classes is not spatially segregated within the AL. Response patterns to the main trailpheromone component nerolic acid remained stable over a wide range of intensities (7-8 log units), while response durations increased indicating that odor quality is maintained by a stable pattern and intensity is mainly encoded in response durations. The structure-function analyses contribute new insights into important aspects of odor processing in a highly advanced insect olfactory system.
\end{abstract}

Indexing terms: glomeruli; 3D-reconstruction; calcium imaging; projection neurons; mushroom bodies; lateral horn; insect brain; antenno-cerebral tract

\begin{abstract}
Odors play an essential role for the regulation of social interactions and colony organization in social insects like ants or bees. Ants heavily depend on chemical communication and species-specific pheromones are essential to organize social behavior (Hölldobler and Wilson, 1990). For example, trail-following behavior, alertness, recruitment, or signaling of the reproductive state are coordinated by the action of pheromones (Hölldobler, 1995). In addition, substances on the body surface (cuticular hydrocarbons) serve as intra- and interspecific recognition cues affecting nestmate recognition as well as intra- and interspecies aggressive interactions (e.g., Singer, 1998; Lenoir et al., 1999; Lahav et al., 1999). Besides signals used for
\end{abstract}

This article includes Supplementary Material available via the Internet at http://www.interscience.wiley.com/jpages/0021-9967/suppmat.

Grant sponsor: German Science Foundation DFG; Grant number: SFB 554 (A6 and A8); Grant sponsor: Evangelisches Studienwerk e.V. Villigst.

*Correspondence to: Wolfgang Rössler, Department of Behavioral Physiology and Sociobiology, Zoology II, Biozentrum, University of Würzburg, Am Hubland, 97074 Würzburg, Germany.

E-mail: roessler@biozentrum.uni-wuerzburg.de 
species-specific communication, a large variety of environmental odors play an important role for orientation and the location and evaluation of food sources (Hölldobler and Wilson, 1990). Thus, the detection, processing, and recognition of a remarkable number of chemical cues by the olfactory system are essential for the survival and reproductive success of ant colonies. These chemosensory tasks require sophisticated sensory machinery and advanced olfactory neuronal networks in the brain of each individual.

In insects the antennal lobes (ALs) in the brain are the first relay station for processing of olfactory information received by olfactory receptor neurons (ORNs) housed in sensilla on the antennae, the main olfactory receptor organs. ORN axons terminate in olfactory glomeruli within the AL. Glomeruli are spheroidal regions of dense synaptic neuropil and in most systems studied so far glomeruli can be regarded as functional units in odor processing (e.g., Hildebrand and Shepherd, 1997; Hansson and Anton, 2000; Galizia and Menzel, 2001; Sandoz, 2006). Molecular and tracing studies have shown that in both vertebrates and insects axons from ORNs that express the same odorant receptor or that have a similar response profile converge on the same glomeruli (Vassar et al., 1994; Mombaerts et al., 1996; Rössler et al., 1999a,b; Vosshall et al., 2000; Xu et al., 2000; Carlsson et al., 2002; Wang et al., 2003). After local processing by a network of local interneurons, olfactory information is transferred via projection neurons (PNs) to higher integration centers in the protocerebrum, the mushroom bodies (MBs), and olfactory neuropils in the lateral protocerebral lobe (LPL), most prominently within the lateral horn $(\mathrm{LH})$. In the honeybee the output from glomeruli is relayed in a distinct pattern to the $\mathrm{MB}$ and $\mathrm{LH}$ via two prominent antennocerebral tracts (medial [m] and lateral [1] ACT) formed mainly by uniglomerular PNs and three small mediolateral ACTs to the LPL mainly formed by multiglomerular PNs (Abel et al., 2001; Müller et al., 2002; Kirschner et al., 2006). Most important, the dendritic fields of the $\mathrm{m}$ - and l-ACT PNs are separated, forming two hemispheres of glomeruli in the AL. Their target areas in the MBs and LH also remain largely segregated, indicating that olfactory information from glomeruli in two $\mathrm{AL}$ hemispheres is transferred and processed via two separate uniglomerular PN output channels (Kirschner et al., 2006).

The functional aspects of odor processing in the hymenopteran brain has been mainly approached by in vivo optical imaging studies in the honeybee showing that within the AL different odors elicit odor specific glomerular activation patterns depending on odor properties such as molecule identity, intensity, and the composition of mixtures (Joerges et al., 1997; Galizia et al., 1999b; Sachse et al., 1999; Carlsson et al., 2002; Carlsson and Hansson, 2003; Sachse and Galizia, 2003; Sandoz, 2003, 2006; Peele et al., 2006). Despite the great importance of olfaction in ants, up to now only one imaging study was carried out (Galizia et al., 1999a), and structural data on the detailed organization of the olfactory pathway is rather limited (Gronenberg, 1999, 2001; Hoyer et al., 2005; Kleineidam et al., 2005).

Research on pheromone processing in insects has largely focused on sex pheromones, especially in moths (e.g., Christensen and Hildebrand 1987; Hildebrand and Shepherd, 1997), where sex-pheromone-specific glomeruli form a macroglomerular complex at the entrance of the male AL (e.g., Hansson et al., 1991; Vickers et al., 1998; Hansson and Anton, 2000; Rospars and Hildebrand, 2000). Sex-pheromone-specific macroglomeruli were also described in the male cockroach and in the honeybee drone (Malun et al., 1993; Brockmann and Brückner, 2001; Sandoz, 2006). A study in leaf-cutting ants revealed a macroglomerulus in large workers of Atta sexdens and A. vollenweideri obviously not associated with sexpheromone processing (Kleineidam et al., 2005). Interestingly, the macroglomerulus was absent in small workers, indicating caste-specific differences in $\mathrm{AL}$ organization in leaf-cutting ants.

Only a few studies have investigated processing of nonsexual pheromones in insects. In social insects nonsexual pheromones are essential for colony organization and survival. Calcium-imaging studies in the carpenter ant $\mathrm{Cam}$ ponotus rufipes and in the honeybee did not reveal a specific clustering of specialized glomeruli responsive to, e.g., alarm pheromone, and the same glomeruli or directly neighboring glomeruli were shown to participate in responses to nonpheromonal odors (Galizia et al., 1999b,c; Sachse et al., 1999; Sandoz, 2006). A recent electrophysiological study in ants showed that alarm-pheromone responsive PNs innervate a specific cluster of normally sized glomeruli within the $\mathrm{AL}$, indicating at least some degree of anatomical segregation of pheromone processing in the ant AL (Yamagata et al., 2006).

The focus of the present study was to ask whether the olfactory system of the highly olfactory carpenter ant, Camponotus floridanus, expresses specific neuroanatomical and/or neurophysiological specializations according to its elaborated role in dealing with a large number of pheromonal and nonpheromonal odors. In particular, we wanted to test:

1) whether the general organization, the number, and the input-output connections of olfactory glomeruli in the AL differ from those found in the well-investigated honeybee (Galizia et al., 1999b; Kirschner et al., 2006);

2) whether the AL has segregated regions for processing pheromonal and nonpheromonal odors; and

3) whether the ant olfactory system is specialized to detect minimal quantities of pheromonal signals and, at the same time, is able to respond appropriately to large ranges of intensities as, for example, required for trail pheromone detection.

To answer the first question we used fluorescent tracing techniques, confocal microscopy, and $3 \mathrm{D}$ reconstructions to analyze the anatomical organization of $\mathrm{AL}$ glomeruli, their antennal sensory input, and their protocerebral output connections. To answer the second and third questions we retrogradely loaded $\mathrm{PNs}$ with a calcium-sensitive dye (Fura-2 dextran) and performed fluorimetric calciumimaging of odor responses of the glomerular (PN) output in the AL. For the third question we focused on intensity coding in response to nerolic acid, a major trail pheromone component in C. floridanus.

\section{MATERIALS AND METHODS}

\section{Animals}

Workers of the carpenter ant, C. floridanus, were used for the experiments. Ants were taken from a colony with a singly mated founding queen collected in Orchid Island, 
FL. Only large workers (head width $>3 \mathrm{~mm}$, body length $\approx 10 \mathrm{~mm}$ ) were used for both neuroanatomical and neurophysiological experiments. The colony was reared under constant conditions in an environmental chamber at $25^{\circ} \mathrm{C}$ and $85 \%$ humidity on a $12 / 12$-h photoperiod. Animals were fed twice a week with honey water, bathkar, and dead cockroaches. Fresh water was given once a week. Ants were anesthetized with $\mathrm{CO}_{2}$ and prepared for anatomical and physiological experiments as described below.

\section{Neuroanatomical procedures}

Whole-mount preparations. For visualization of brain structures, especially AL glomeruli, we utilized tissue autofluorescence caused by glutaraldehyde fixation. The ants were decapitated and the head capsule fixed in dental wax-coated dishes. The head capsule was opened by cutting a window between the compound eyes and the brain was rinsed immediately using fresh ant-saline solution $\left(127 \mathrm{mM} \mathrm{NaCl}, 7 \mathrm{mM} \mathrm{KCl}, 1.5 \mathrm{mM} \mathrm{CaCl}_{2}, 0.8 \mathrm{mM}\right.$ $\mathrm{Na}_{2} \mathrm{HPO}_{4}, 0.4 \mathrm{mM} \mathrm{KH}_{2} \mathrm{PO}_{4}, 4.8 \mathrm{mM}$ TES, $3.2 \mathrm{mM}$ Trehalose, $\mathrm{pH}$ 7.0). Glands and tracheae were removed and the brains were dissected out and fixed immediately in cold $1 \%$ glutaraldehyde in phosphate-buffered saline (PBS, $\mathrm{pH}$ 7.2 ) for 4 days at $4^{\circ} \mathrm{C}$. The brains were then washed in PBS $(5 \times 10$ minutes $)$ and dehydrated in an ascending series of ethanol $(30 \%, 50 \%, 70 \%, 90 \%, 95 \%, 3 \times 100 \%, 10$ minutes each step). Finally, the brains were cleared in methylsalicylate (M-2047, Sigma Aldrich, Steinheim, Germany) and mounted in special aluminum slides with a central hole covered by thin coverslips from both sides. Whole-mounts were stored at $-20^{\circ} \mathrm{C}$.

Tracer application and tissue preparation. For antennal backfill preparations, all animals were fixed in customized acrylic blocks and the heads and antennae were stabilized with dental wax. Both antennae were cut at the lower part of the pedicellus. The cut surface was immediately covered with a drop of dextran-biotin (D7135, Molecular Probes, Eugene, OR) dissolved in distilled water, and the preparation was kept in a moistened chamber overnight to let the dye diffuse. The brains were then dissected and fixed immediately in $4 \%$ formaldehyde in $0.1 \mathrm{M}$ PBS overnight at $4^{\circ} \mathrm{C}$. After washing with $0.1 \mathrm{M}$ PBS ( $3 \times 10$ minutes) the brains were incubated in Alexa 568-conjugated streptavidin (S-11223, Molecular Probes) in PBS with $0.2 \%$ Triton-X (1:250), first for 1 hour at room temperature, then overnight at $4^{\circ} \mathrm{C}$. Finally, the brains were washed with $0.1 \mathrm{M}$ PBS $(4 \times 5$ minutes on a shaker at room temperature), dehydrated in an ascending alcohol series $(30 \%, 50 \%, 70 \%, 90 \%, 95 \%, 3 \times 100 \%, 10$ minutes each step) and cleared in methylsalicylate and mounted in permount (Fischer Scientific, Schwerte, Germany).

For labeling of the ACTs the ants were fixed in dental wax dishes. The heads were fixed in an anterior and posterior preparation to access the front or back of the brain depending on the area of dye application. In either case a cut between the compound eyes was made to open the head capsule and glands and tracheae were removed. The tracer was applied into different brain regions to selectively stain the tracts of interest (Kirschner et al., 2006). Two dextran conjugates were used: rhodamine dextran with biotin (3,000 MW, lysine-fixable; Microruby, D 7162, Molecular Probes) and Alexa Fluor 488 dextran (10,000 MW, lysine-fixable; D 22910, Molecular Probes). The tracers were applied as follows: glass micropipettes were pulled with a horizontal laser-electrode puller
(P2000, Sutter Instruments, Novato, CA) using borosilicate capillaries (1B100F-3, Precision Instruments, Sarasota, FL). The broken tip of each glass electrode was coated with small dye crystals $(\approx 200 \mu \mathrm{m}$ in diameter). Prior to inserting the dye-coated pipettes into brain tissue the region of interest was carefully perforated with an unbroken glass pipette. Subsequently, the dye electrode was plunged into the perforated area and remained for up to 10 seconds in the target area. The pipette was removed and the brain immediately rinsed with fresh ant-ringer solution to wash out excessive dye. To investigate the AL connections within the protocerebrum, dye was inserted during the anterior preparation directly into the AL neuropil. For double labeling of the projection areas of the medial and lateral ACT in the MBs and $\mathrm{LH}$ we used a technique described by Kirschner et al. (2006), but accessing the ant brain from posterior. The m-ACT was labeled by insertion of the dextran tracer in the medial-caudal protocerebrum and for the l-ACT the dextran tracer was placed into the lateral-caudal protocerebrum. To doublelabel ORN axons and the dendritic arborizations of PNs in the AL we combined anterograde ORN mass fills with retrograde mass fills of $\mathrm{m}$ - and $\mathrm{l}-\mathrm{ACT} \mathrm{PNs}$ in a posterior preparation. Briefly, one antenna was cut at the base of the scapus and dextran-conjugated fluorescent dye was immediately applied at the cut surface. In a second step either the m- or l-ACT was counterstained using the method described above.

\section{Confocal laser-scanning microscopy and 3D- reconstructions of glomeruli and tracts}

All brains were viewed as whole-mount preparations using two confocal laser scanning microscopes (Leica TCS LP and Leica TCS SP2 AOBS; Leica Microsystems, Wetzlar, Germany) equipped with an argon/krypton and helium/neon laser. Excitation wavelengths were $568 \mathrm{~nm}$ for rhodamine and streptavidin-conjugated Alexa 568 and $488 \mathrm{~nm}$ for Alexa Fluor 488. Two different HC PL APO objective lenses were used for image acquisition $(10 \times 0.4$ $\mathrm{NA}$ imm and $20 \times 0.7 \mathrm{NA} \mathrm{imm)} \mathrm{and} \mathrm{optical} \mathrm{sections} \mathrm{were}$ taken at distances between 1-10 $\mu \mathrm{m}$. In certain cases a digital zoom of $2-3 \times$ was applied. Double-labeled specimens were scanned sequentially. All confocal image stacks were viewed and processed with the 3Dreconstruction software AMIRA 3.1 (Mercury Computer Systems, Berlin, Germany). To obtain a better signal-tonoise ratio some preparations were deconvoluted using the AMIRA deconvolution algorithm. In doubly labeled preparations each channel was set to a false color. Images were scaled and snapshots were taken from single optical sections or complete stacks. Screenshots were further processed in Adobe Photoshop 6.0 and 7.0 software (Adobe Systems San Jose, CA) and adjusted for brightness and contrast. Anatomical directions refer to Strausfeld (2002) and Kirschner et al. (2006). Single glomeruli were clearly visible as densely packed neuropil structures and were outlined in all focal planes $(\mathrm{yz}, \mathrm{xy}, \mathrm{xz})$. 3D-reconstructions of individual glomeruli were done using the Amira 3.1 feature "wrap." Antennal sensory input tracts as well as AL output tracts (and somata) were rendered using the "interpolate" and "automatic threshold" feature. Individual glomeruli or cluster of glomeruli associated with the same antennal sensory input tracts (T1-T7) were color coded. 


\section{Preparation and dye loading for calcium imaging}

Ants were briefly (for a few seconds) anesthetized with $\mathrm{CO}_{2}$ and fixed in a Plexiglas stage using soft dental wax (surgident periphery wax, Heraeus Kulzer, Germany). Compared to treatment with $\mathrm{CO}_{2}$, anesthetization by cooling on ice did not show any obvious differences in the responses to the odors tested (see below). A small window was cut into the head capsule to access the ventral part of the brain and the site for dye application. A sharp glass electrode coated with a few crystals of Fura-2 dextran (potassium salt, 10,000 MW, F3029, Molecular Probes) dissolved in $2 \%$ bovine serum albumin solution was inserted for several seconds into the lateral protocerebrum, dorsolaterally to the vertical lobe of the MBs, aiming for the m- and l-ACT (see method above and Fig. 2F,G). Following dye application the brain was rinsed with antsaline solution to remove excess dye. The window in the head capsule was closed with the cut piece of cuticle and the ants were released from the Plexiglas stage for 4 hours. During the staining period the ants were allowed to move freely in a moistened Plexiglas container before they were fixed again in the Plexiglas stage as before. Antennae were immobilized with wax and a larger window was cut into the head capsule to access the whole brain and the ALs. Glands and tracheae were carefully removed and the esophagus was cut at the mouth parts and pulled out of the head capsule to prevent movement of the brain during data acquisition. We prepared a total of 307 ants for Fura-2 dextran labeling and calcium imaging; 145 (47\%) resulted in bright staining in the ALs, but $82(\approx 27 \%)$ of the ants with staining in the ALs showed no spontaneous activity or responses to odor stimulation. In 34 ants $(\approx 11 \%)$ clear spontaneous activity was observed, but no responses to odor stimulation. We therefore used 29 ants $(\approx 9-10 \%$ of the total number $)$ that were clearly stained, showed spontaneous activity, and clear responses to odor stimulation for further analyses. We were able to test the complete stimulation program including all odor intensities in seven $(\approx 2 \%)$ of these 29 ants (see below).

\section{Imaging}

Calcium-imaging experiments were performed using an Olympus imaging system (Cell, v. 1.1-2.3) with an upright epifluorescent microscope (BX51WI; with the filter set UM2FUR) equipped with an LD $20 \times$ water-immersion lens (XLUMP, NA 0.95) and epifluorescent illumination was provided by a $150 \mathrm{~W}$ xenon light source (MT20, with excitation filters for $340 \mathrm{~nm}$ and $380 \mathrm{~nm}$ ). The focal plane within the AL was adjusted to a depth of $35 \mu \mathrm{m}$ below the surface of the AL using a piezo-driven nanofocusing system (PIFOC, PI, Germany). For each stimulus a series of 24 double frames was recorded with an air-cooled CCD camera (model 8484-03G, Hamamatsu Photonics, Japan) at a sampling rate of $4 \mathrm{~Hz}$. A $2 \times 2$ on chip binning resulted in an image pixel size of $0.645 \times 0.645 \mu \mathrm{m}$. Exposure times ranged from $40-60 \mathrm{~ms}$ for the first frame at $340 \mathrm{~nm}$ and $20-30 \mathrm{~ms}$ for the second frame at $380 \mathrm{~nm}$. Odor stimulation started at frame 10 and was terminated at frame 14 (1 second). Repeated odor stimuli were given at an interstimulus time interval of at least 1 minute.

Imaging data were analyzed by calculating the ratio of fluorescence intensity in the images taken at 340 and 380 $\mathrm{nm}$ excitation for each pair as: $\mathrm{R}=\mathrm{F}_{340} / \mathrm{F}_{380}$, and subse- quently applying an $\mathrm{N} \times \mathrm{N}$ filter $(5 \times 5$ pixels with 5 iterations) to reduce noise. Autofluorescence and stained neurons caused inhomogeneous fluorescence images (background fluorescence) and by subtracting the average ratio-image using frames 1 to 9 from all ratio images the background was set to zero prior to odor stimulation. Filtered ratio-images with background subtraction are labeled as $\Delta\left(\mathrm{F}_{340} / \mathrm{F}_{380}\right)$.

Following odor stimulation, calcium signals were measured as changes in fluorescence $\Delta\left(\mathrm{F}_{340} / \mathrm{F}_{380}\right)$ and considered as neuronal activity in response to a given odor stimulus when they exceeded $40 \%$ of the maximal response. The maximal response was measured across all different odors and concentrations tested in the preparation. Neuronal activities (activity areas) are represented as false-color-coded images using the average of frame 11 to 14 (during odor stimulation). In most cases, activity areas could not be assigned to single glomeruli because often their size was considerably larger than the size of single glomeruli. The circular shape and small size (10-40 $\mu \mathrm{m})$ of other activity areas were similar to the dimensions of single glomeruli and termed activity spots.

The spatial overlap of activity areas in response to two odors was calculated as the percentage of an odor specific activity area that was activated by the other odor. All pixels within the AL with intensity values above threshold (40\% of the maximal response) during stimulation with one odor were counted and compared with the odorspecific activity areas elicited by one of four other odors using the software AMIRA 3.1 (Mercury Computer Systems, Germany). Calcium signals in a total of five animals and in response to five different odors were used for this analysis.

For four different odors, threshold odor concentrations were measured in 4-11 animals using the threshold criterion mentioned above. In order to analyze the dynamic range of the calcium responses at the different odor concentrations, at least two areas with the highest calciumsignal amplitudes (circular regions of interest, ROIs) were selected and normalized within each animal. The calciumsignal amplitude within the ROIs and the duration (number of frames) was measured across all tested odor concentrations (dilutions: $10^{-11}, 10^{-8}, 10^{-5}$, and $10^{-2}$ ). To analyze whether signal amplitude and duration correlate with odor intensity, two ROIs of each preparation were separated according to either showing clear concentration-independent calcium-signal amplitudes $(\mathrm{cd}-)$ or concentration-dependent calcium-signal amplitudes $(\mathrm{cd}+)$. Signal durations within the resulting two classes of ROIs were compared using a Spearman'srank correlation on pooled data of seven animals.

\section{Odor stimulation}

A constant and moistened air stream of $1 \mathrm{~L} / \mathrm{min}$ was produced by two independent flow controllers (VC-2LPM, Alicat Scientific, Tucson, AZ), both set to $0.5 \mathrm{~L} / \mathrm{min}$ and connected to two solenoid valves controlled by the imaging software. The solenoid valves allowed switching of each of the two flow channels through a plastic cartridge $(1 \mathrm{~mL})$ containing a filter paper $\left(1 \mathrm{~cm}^{2}\right)$. Only one of the two flow channels was used in the experiments. For odor stimulation, $5 \mu \mathrm{L}$ of the odor diluted in mineral oil (Sigma Aldrich, Deisenhofen, Germany) was applied onto the filter paper and the cartridge was placed into the olfactometer. Odor dilutions ranged from $10^{-1}$ to $10^{-12}$ and experiments al- 
ways started with the lowest odor concentration. As control stimulus, $5 \mu \mathrm{L}$ of mineral oil was applied onto the filter paper.

Eight different odors including two pheromones were used for stimulation. As general odors, citral, isoamylacetate (IAA), nerol, 1-hexanol, heptanal, and 1-octanol (all from Sigma Aldrich) were used. As pheromones of $C$. floridanus, the alarm pheromone, $\mathrm{n}$-undecane (Sigma Aldrich) and the trail pheromone (releaser component), nerolic acid (Haak et al., 1996; Cardiff Chemicals, Cardiff, UK) were used for odor stimulation.

Activity areas were described for all odors, but not all odors were tested in each experiment. Odor representation across animals was investigated using heptanal, isoamylacetate, citral, and nerolic acid. The overlap of activity areas in response to different odors and the threshold concentration for each single odor were investigated using nerolic acid, n-undecane, citral, heptanal, and 1-octanol. The duration of the calcium signal across different odor concentrations was investigated for the known trail pheromone component nerolic acid.

\section{RESULTS}

\section{Sensory-tract innervation of glomeruli}

The mass-labeled projections of ORN axons were analyzed to investigate the pattern of sensory-tract-specific innervation of glomerular clusters in the $\mathrm{AL}$ of $C$. floridanus $(\mathrm{n}=6)$. A representative example is shown in Figure 1 and the corresponding 3D-reconstruction is shown in Figure 2B,C (see also Suppl. 3D online material). The complete brain reconstruction in Figure 2A shows that $C$. floridanus has small optic ganglia and relatively large ALs underlining the mainly olfactory sensory orientation of this ant. In the AL of six preparations we counted between 434 and 464 glomeruli, indicating some variability in the total number of glomeruli in large workers. The mean number of glomeruli was $452 \pm 14$. Tracing of the antennal nerve (AN) revealed that ORN axons separate (together with axons from motor neurons and mechanoreceptors that proceed further to the dorsal lobe, DL) forming seven distinct sensory tracts that enter the AL (T1-T7). Each tract innervated a characteristic cluster of glomeruli dividing the AL into seven glomerular subregions. In the following, these glomerular clusters are termed T1-7 clusters (Figs. 1, 2B,C). An overview of the number of glomeruli innervated by T1-7 together with their volumes and ACT associations are given in Table 1 . The tracts were identified by closely following their trajectories image by image in 3D image stacks (see example of an image stack in Suppl. material to Fig. 1, and a rotating image of the 3D reconstruction in Suppl. materials to Fig. 2). T1 proceeded at the ventral surface of the AL and innervated a small cluster of $\approx 34$ glomeruli in the ventral-rostral part of the AL (Figs. 1A,B, 2B; Table 1). This cluster was flanked laterally by the T2 and medially by the T3 cluster. The T2 cluster extended along the ventral-lateral side of the AL and consisted of $\approx 56$ glomeruli. The T3 cluster with 96 glomeruli spread along the ventral medial part of the AL. Since the clear separation of T3 and T5 glomeruli was most difficult, we used additional criteria to distinguish among the two clusters such as the smaller size of T3 glomeruli compared to T5 glomeruli. A group of $\approx 78$ large glomeruli comprised the T4 cluster at the dorsal-lateral side of the AL. The relatively large T4 proceeded transversally through the inner lateral part of the AL (Figs. $1 \mathrm{C}, \mathrm{D}, 2 \mathrm{C})$. Opposite to this cluster, in the dorsal-medial $\mathrm{AL}, \approx 36$ glomeruli were innervated by $\mathrm{T} 5$, which proceeded transversally through the AL in a dorsal-medial direction. Next to the T5 cluster we found the largest group of glomeruli in the AL, the T6 cluster, consisting of $\approx 128$ comparatively small glomeruli (Table 1 ; Figs. $1 \mathrm{~B}-\mathrm{D}$, $2 \mathrm{C})$. The T6 proceeded along the dorsal-medial surface of the $\mathrm{AL}$ and entered its target glomeruli from the periphery. The smallest cluster of T7 glomeruli was located in the dorsal part of the AL, close to the DL, and consisted of six comparatively large glomeruli. The volume of these glomeruli was calculated as 4-10 times larger than that of most other AL glomeruli, and their innervation was characterized by particularly brightly labeled bundles of axons (Figs. 1D, 2C, thin dashed lines; Table 1; and Suppl. 3D online material). In all preparations these glomeruli were stained brightly. The corresponding ORN axons reached the T7 glomeruli via several loose bundles which we term the $\mathrm{T} 7$ tract.

\section{Hemispherical division of AL glomeruli by m- and l-ACT output}

Two prominent AL output tracts, the m- and l-ACT, were successfully double-labeled in two preparations and the m-ACT was selectively labeled in four cases, the l-ACT in three cases. One example of a doubly labeled AL and the corresponding 3D-reconstruction of glomeruli supplied by the m-ACT (magenta) and l-ACT (green) are shown in Figure 2D,E. Please note that the colors in 2D,E do not reflect connectivity with glomeruli with similar colors in Fig. 2B (for a summary of the input-output connectivity, see Table 1). Our recent study in the honeybee revealed a similar hemispherical division of AL glomeruli (Kirschner et al., 2006). Compared to the situation in the honeybee, however, the two AL hemispheres in $C$. floridanus contained a much higher number of glomeruli (Table 1) and appeared rotated by $\approx 45^{\circ}$ along the anterior-posterior and medio-lateral axes. In addition to the m- and l-ACT, and similar to the situation described in the honeybee, three small ml-ACTs (1-3) projected directly to the lateral horn (LH), and two of them (ml-ACT 2 and 3) formed a "lateral network" between the mushroom body (MB) vertical lobe and the LH (Fig. 2F,G).

In the example shown, a total of 216 glomeruli in the rostral-ventral hemisphere of the $\mathrm{AL}$ were innervated by PNs of the 1-ACT (green; Fig. 2D,E; Table 1). The l-ACT axons ran dorsally through the inner nonglomerular core of the AL. They formed a single prominent bundle leaving the $\mathrm{AL}$ in dorsal direction, bending rostral-laterally and projecting to the ipsilateral $\mathrm{LH}$ before innervating the MB calyces (Fig. 2E-G). The somata of l-ACT PNs were arranged in two distinct clusters (1SC): a large ventral cluster on the l-ACT hemisphere (ISC1, Fig. 2E) and a smaller cluster along the rostral lateral rim of the l-ACT hemisphere (ISC 2; visible in 3D Suppl. material). A total of 218 glomeruli in the caudal-dorsal AL hemisphere were innervated by m-ACT PNs (magenta) (Table 1; Fig. 2D,E). The axons of $\mathrm{m}$-ACT PNs projected rostrally through the nonglomerular AL neuropil and formed two separate bundles. These bundles merged after passing the l-ACT-bundle, but before exiting the AL to form the m-ACT (Fig. 2F,G). Somata of the m-ACT (mSC) were arranged in two large 

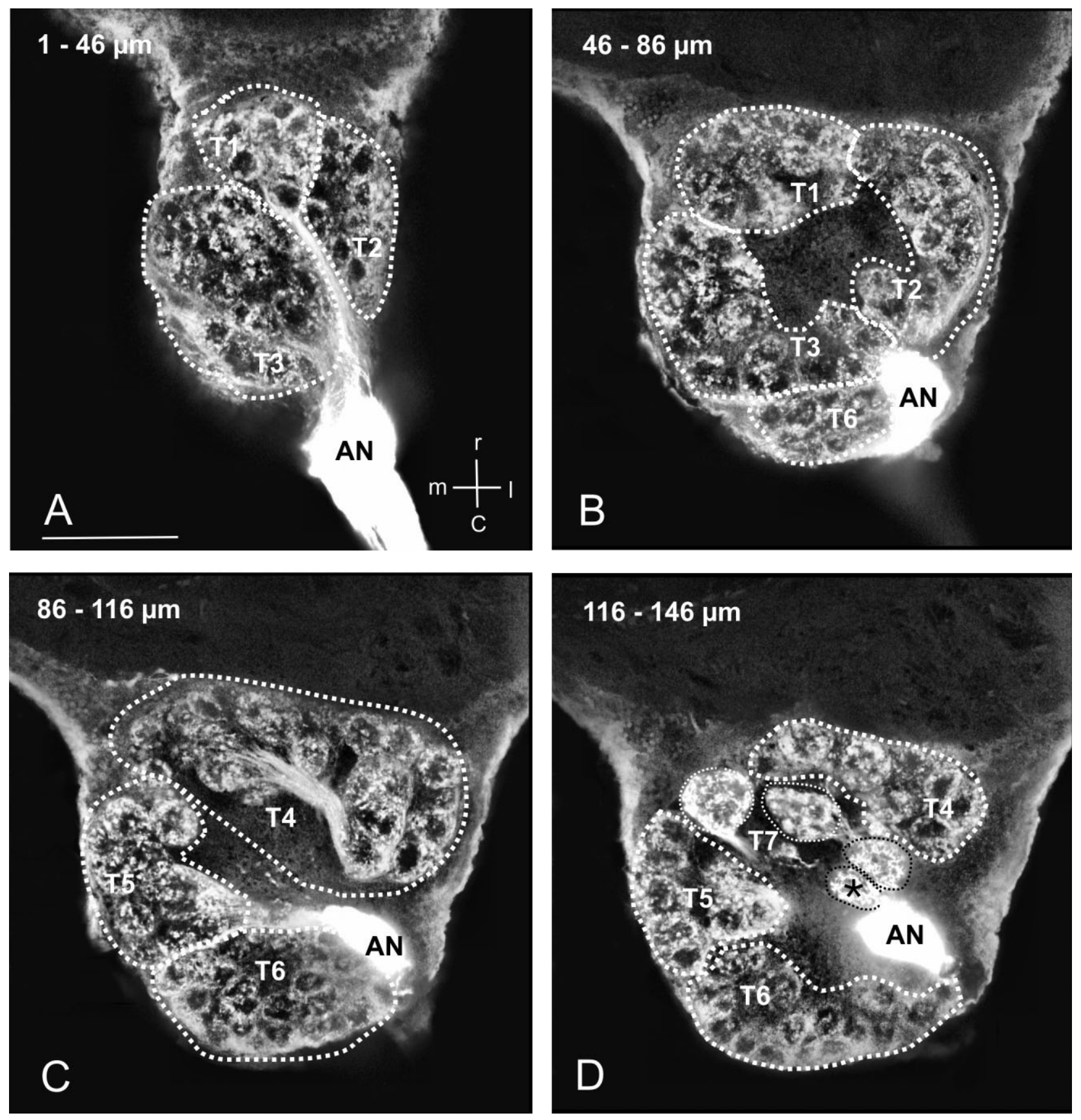

Fig. 1. Antennal sensory innervation of the antennal lobe (AL). A-D: Series of confocal images of an AL with an anterogradely labeled antennal nerve showing the sensory innervation of AL glomeruli via seven distinct antennal sensory tracts (T1-T7) and the associated cluster of glomeruli. Single optical sections at different depths (indicated in $\mathrm{A}-\mathrm{D}$ ) show the seven tracts and parts of the innervated

glomerular clusters (for further details, see complete image stack in Suppl. materials). The asterisk in D labels the single T7 glomerulus that is not innervated by m- or l-ACT PNs. Spatial directions indicated in A: rostral, r; caudal, c; medial, m; lateral, l. Scale bar $=100$ $\mu \mathrm{m}$ in A (applies to all).

clusters, one triangle-shaped cluster in the rostral medial region $(\mathrm{mSC} 1)$ and a smaller band of somata in the caudal lateral part of the $\mathrm{AL}(\mathrm{mSC} 2$, Fig. $2 \mathrm{E})$. With the exception of one glomerulus, all glomeruli within the AL could be assigned to either the m- or l-ACT, and none of them was innervated by both (Table 1). The one glomerulus that was never found labeled after $\mathrm{m}$ - or l-ACT

staining belonged to the dorsal-rostral T7 cluster (asterisk in Fig. 1D). It showed similarities regarding its position with glomerulus D02 in the honeybee (Kirschner et al., 2006). The relatively small variance in the total number of glomeruli ( \pm 14 , see results above) indicates that variance in the input-output connectivity in the seven clusters is rather low. 


\section{Afferent and efferent connection of $\mathrm{AL}$ glomeruli}

To assign the antennal sensory tracts to either the m- or l-ACT we combined anterograde ORN mass-fills with either retrograde labeling of the $\mathrm{m}-\mathrm{ACT}$ (two preparations) or the l-ACT (three preparations). All preparations revealed a clear distribution of six of the seven tracts plus associated glomeruli cluster to either the m- or l-ACT hemisphere. T1, T2, and T4 exclusively belonged to the l-ACT hemisphere (Table 1). T5 and T6 were innervated by m-ACT PNs. Similarly, the T7 cluster was innervated by m-ACT PNs, except for one large glomerulus, which was neither innervated by m- nor l-ACT PNs. The glomeruli of the T3 cluster could be divided into two about equally large subgroups innervated by either the $\mathrm{m}$ - or l-ACT. The upper rostral part belonged to the l-ACT hemisphere, the caudal part to the m-ACT hemisphere. A similar innervation pattern was recently shown for the T3a cluster in the honeybee (Kirschner et al., 2006).

\section{Segregated olfactory input to the MB calyces and lateral protocerebral lobe}

Olfactory information from the AL is transferred to the MB calyces via the $\mathrm{m}$ - and l-ACT. Their course through the protocerebral lobe is shown in the projection view in Figure $2 \mathrm{~F}$ and a $3 \mathrm{D}$ visualization of their course is shown in Figure 2G. Double staining of the $\mathrm{m}$ - and l-ACT projections in the same preparation $(n=1)$ revealed a prominent innervation of the lip region (Fig. $2 \mathrm{H}$ ). This pattern was confirmed by single stainings of either the $\mathrm{m}-(\mathrm{n}=3)$ or l-ACT $(\mathrm{n}=4)$. The collar region was not innervated by m- or l-ACT PNs. Throughout the basal part and the upper part of the lip only the peripheral part was innervated by $\mathrm{PNs}$ of the $\mathrm{m}-\mathrm{ACT}$, whereas the inner part was innervated by the l-ACT. The lip region appeared subdivided into three concentric layers (Fig. 2H; I, II, and III). The inner core and the intermediate layer of the lip were predominantly innervated by the l-ACT and only few boutons of m-ACT PNs were found. Compared to the inner core, the intermediate layer of the lip showed a higher density of boutons. In contrast, the outer rim of the lip region was most densely packed with boutons, exclusively from m-ACT PNs.

Innervation of the $\mathrm{LH}$ was compartmentalized into predominantly m- or l-ACT domains (Fig. 3) $(\mathrm{n}=5$ preparations). Figure $3 \mathrm{~A}$ combines results from two different preparations in two different ants. Figure 3A shows one preparation with both $\mathrm{m}$ - and l-ACT stained and the $\mathrm{m}$-ACT innervation was analyzed and highlighted in the LH. Figure $3 \mathrm{~B}$ shows a second preparation in which the m-ACT was cut to prevent the dye from diffusion to the m-ACT target regions in the LH. In this case only l- and ml-ACT fibers were stained, and their innervation pattern in the LH shows clear separation from the m-ACT target region. The axons terminated in a club-shaped medial compartment of the $\mathrm{LH}$, which was exclusively innervated by m-ACT PNs (Fig. 3A; dashed line). The closely surrounding neuropil contained only a few m-ACT innervation, but dense branches of l-ACT and ml-ACT PNs $(\mathrm{n}=4)$ (Fig. 3B). The data indicate a zone of overlap of $1-$ and ml-ACT fibers in an intermediate region within the LH. This pattern indicates a topographical separation of $\mathrm{m}$ and l-ACT input to the LH strikingly similar to that found in the honeybee (Kirschner et al., 2006).
PNs leaving the AL via three ml-ACT showed a distinct projection pattern in the lateral protocerebral lobe (LPL) and LH. The axons of the ml-ACT1 bypassed the LPL and targeted the LH only. The axons and synaptic fields of ml-ACT 2 and 3 formed a characteristic arborization pattern within the LPL similar to the "lateral network" found in the honeybee. Kirschner et al. (2006) describe three distinct olfactory innervation foci in the LPL of the honeybee: the ring neuropil, located ventrally in the brain around the vertical lobe of the MB, the triangle, a small region in the dorsal center of the LPL, and the lateral bridge between the triangle and the LH. In C. floridanus similar innervation foci of ml-ACT axons were found in corresponding regions of the LPL (Fig. 3C,D). Compared to the honeybee, the ring neuropil in $C$. floridanus appeared to extend less around the MB vertical lobe.

\section{Calcium imaging of odor responses in the antennal lobe}

We measured intracellular calcium changes in AL projection neurons (PNs) in response to different odors. Both $\mathrm{m}$ - and l-ACT PNs were retrogradely filled with the calcium-sensitive dye Fura-2 dextran and successful dye uptake resulted in a bright fluorescence of glomeruli and somata clusters in the AL. In all preparations tested spontaneous neuronal activity (changes in calcium amplitude) could be observed in areas that were comparable to single glomeruli in shape and size (activity spots). During odor stimulation, changes in calcium responses were visible in larger areas of the AL (activity areas) or in activity spots. In most cases it was not possible to assign activity areas to particular glomeruli or activity spots to a single glomerulus because optical resolution of the fluorescent images did not allow reliable discrimination of all glomeruli $(\approx 50)$ in the field of view. Therefore, we compared odor representation in the $\mathrm{AL}$ in response to stimulation using activity areas. Glomerulus diameter in the imaged area ranged between 15-20 $\mu \mathrm{m}$ (Fig. 2; volumes in Table 1). Some activity patterns covered larger areas within the $\mathrm{AL}$, indicating that in these cases groups of glomeruli rather than single glomeruli were activated. For measurements (see below), the ROIs were set not larger than $20 \mu \mathrm{m}$ to avoid measurement of such "group" effects.

\section{Odor-specific calcium signals}

In response to stimulation, odor-specific activity areas were found in 39 animals using 2-8 different odors at high concentration (dilution: $10^{-1}$ ). Repeated odor stimulation with the same odor resulted in similar activity areas (data not shown). In response to some odors (citral, isoamylacetate, nerol, and heptanal) the activity areas recorded in individual ants covered large parts of the $\mathrm{AL}$, whereas responses to other odors (1-octanol, 1-hexanol, $\mathrm{n}$-undecane, and nerolic acid) were more confined in a few activity spots (Fig. 4A-F). As an example, odor-specific activity areas measured in one animal are shown as falsecolor-coded images. Pheromone stimulation resulted in qualitatively similar activity areas as compared to general odors (Fig. 4F,K,O,P-U). Odor-specific activity areas were similar across individuals as shown for stimulation with heptanal (dilution: $10^{-1}$ ), isoamylacetate (IAA; dilution: $10^{-1}$ ), citral (dilution: $10^{-1}$ ), and nerolic acid (dilution: $10^{-9}$ ) in two ants (Fig. 4G-O). The activity areas in response to different odors overlapped both within the group of general odors as well as between general odors and 
B
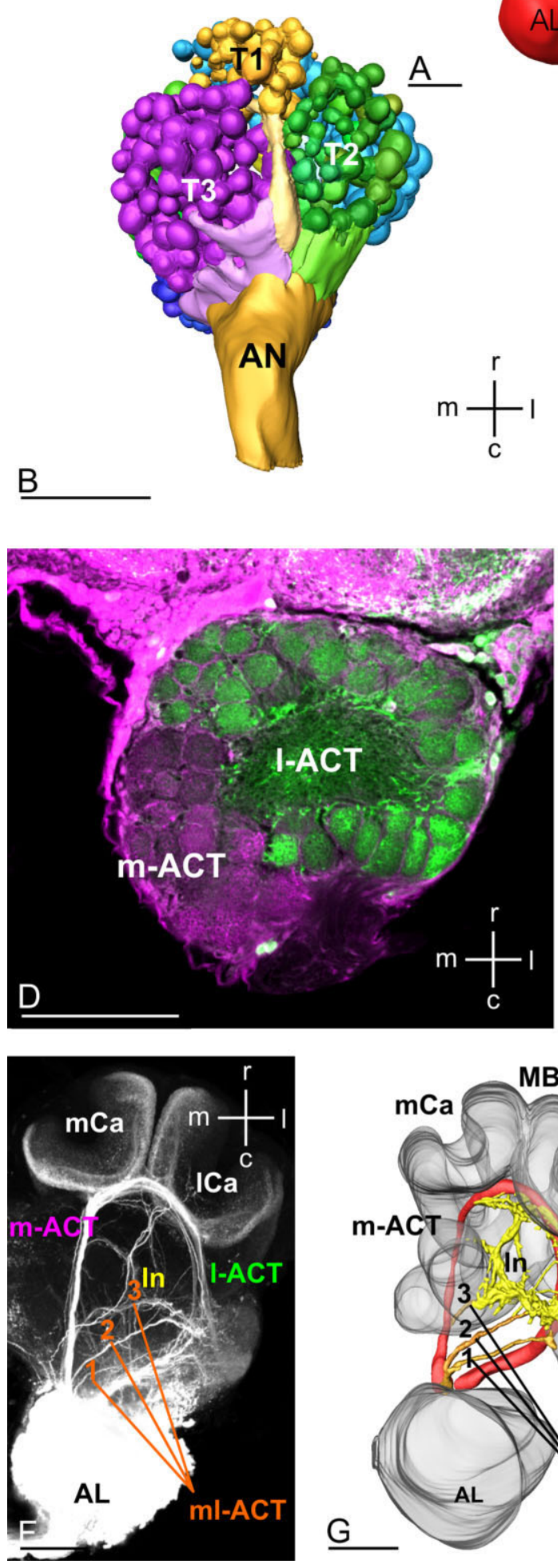

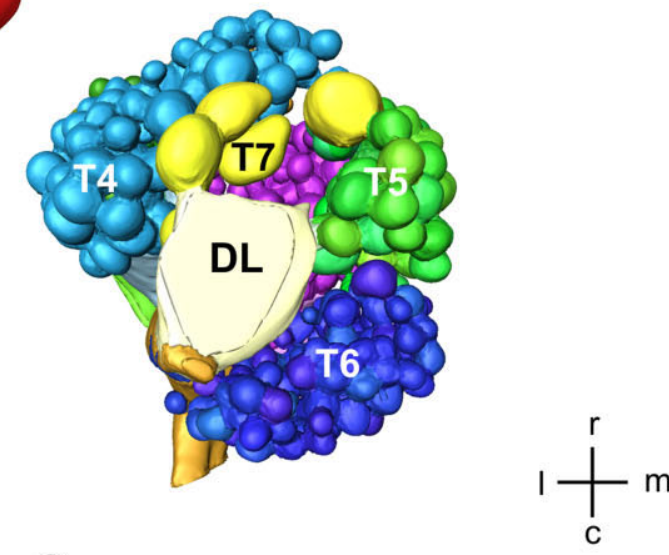

C
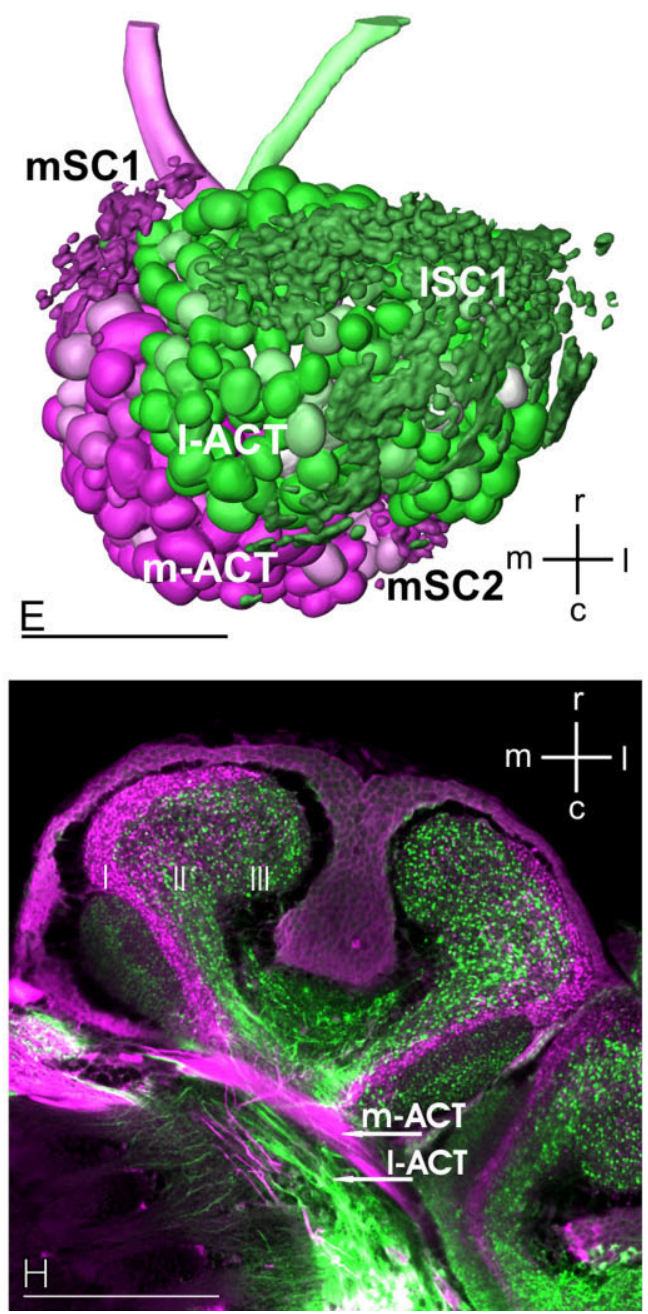
TABLE 1. Summary of the Afferent and Efferent Connection of Glomeruli

\begin{tabular}{|c|c|c|c|c|c|c|c|c|c|}
\hline & & $\mathrm{T} 1$ & $\mathrm{~T} 2$ & T3 & $\mathrm{T} 4$ & T5 & T6 & $\mathrm{T} 7$ & $\Sigma$ \\
\hline A & $\Sigma$ glomeruli & 34 & 56 & 96 & 78 & 36 & 128 & 6 & 434 \\
\hline B & $\underset{\left[\mu \mathrm{m}^{3} \times 10^{3}\right]}{\sum \text { volume }}$ & 91.6 & 159.5 & 402.8 & 423.6 & 255.0 & 331.4 & 133.2 & $1,797.1$ \\
\hline $\mathrm{C}$ & $\begin{array}{l}\text { Max volume } \\
\quad\left[\mu \mathrm{m}^{3} \times 10^{3}\right]\end{array}$ & 10.0 & 8.4 & 9.8 & 19.2 & 12.6 & 7.0 & 41.2 & \\
\hline $\mathrm{D}$ & $\begin{array}{l}\text { Min volume } \\
\qquad\left[\mu \mathrm{m}^{3} \times 10^{3}\right]\end{array}$ & 0.9 & 1.1 & 1.3 & 1.8 & 4.9 & 0.4 & 10.8 & \\
\hline $\mathrm{E}$ & $\begin{array}{l}\text { Mean volume } \\
\qquad\left[\mu \mathrm{m}^{3} \times 10^{3}\right]\end{array}$ & 2.7 & 2.8 & 4.2 & 5.4 & 7.1 & 2.6 & 22.2 & \\
\hline $\mathrm{F}$ & Output tract innervation & l-ACT & 1-ACT & l-ACT/m-ACT & l-ACT & m-ACT & $\mathrm{m}-\mathrm{ACT}$ & $\begin{array}{c}\text { m-ACT } \\
\text { (5 out of } 6)\end{array}$ & \\
\hline
\end{tabular}

A, B: Sum of the number and total volumes (in $\mu \mathrm{m}^{3} \times 10^{3}$ ) of all glomeruli belonging to a cluster supplied by the seven antennal sensory tracts T1-T7. C, D, E: Maximum (Max), minimum (Min) and mean volume of glomeruli belonging to the different tract-specific clusters. F: Affiliation of the different clusters to the two main projection neuron output tracts (m- or l-ACT).

pheromones (estimated areas ranged from very little overlap to up to $\approx 70 \%$ ). The activity areas of the two pheromones overlapped each other in the range of $\approx 10-70 \%$.

\section{Odor concentration threshold for calcium signals}

To investigate the sensitivity of the olfactory system in C. floridanus we measured the lowest odor concentration that elicited consistent calcium patterns in the AL (Fig. 5). We found a very high sensitivity of PN calcium responses to both general odors (1-hexanol, 1-octanol) and pheromones (nerolic acid, n-undecane). In at least $80 \%$ of the investigated animals, activity areas were found in response to very low odor concentrations (dilution: $10^{-11}$ ) of the four odors tested (Fig. 5A). We only tested one odor quality at different concentrations in each animal (total $\mathrm{n}=29$ ), thus, all four response thresholds are independent of each other.

\section{Dependence of odor representation on odor concentration}

The trail pheromone component nerolic acid was used as stimulus to investigate the variance of activity areas and the dynamic range of the calcium activation patterns across odor concentrations (11 log units). In all seven animals investigated we found that the spatial pattern of activity areas in response to nerolic acid were odor- concentration-dependent only at very high and very low concentrations (Fig. 4P-U). The response threshold for nerolic acid was at a dilution of $10^{-11}$ with only a single activity spot. At a dilution of $10^{-9}$, additional activity spots were found (arrows in Fig. 4Q), and this pattern remained largely stable over a concentration range of 7-8 log units (Fig. 4Q-T). At the highest odor concentration tested (dilution: $10^{-1}$ ) a further activity spot added to the existing pattern (Fig. 4U; arrow).

The amplitude of the calcium signals from activity spots was analyzed over different odor concentrations (Fig. 5B). ROIs were defined around activity spots and response amplitudes were normalized within each animal (see Materials and Methods). The amplitude of the calcium signal within the ROIs was concentration-dependent in some cases $\left(\mathrm{ROI}_{\mathrm{cd}+}\right)$ and not concentration-dependent in other cases $\left(\mathrm{ROI}_{\mathrm{cd}-}\right)$. The pairs of ROIs, selected in each preparation of the seven animals investigated, were split to

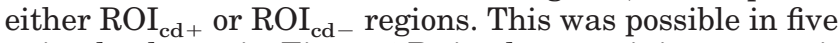
animals shown in Figure 5B; in the remaining two animals no clear concentration dependency was found. To compare the duration of the calcium signal, $\mathrm{ROI}_{\mathrm{cd}+}$ and

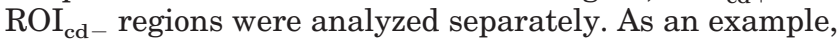
the time courses of the response of one $\mathrm{cd}+$ and one $\mathrm{cd}-$ to three odor concentrations are shown together with the control (response to solvent) in Figure 5C. The response dynamics for all measured ants were further analyzed.
Fig. 2. The olfactory pathway in the brain of Camponotus floridanus. Afferent and efferent innervation of antennal lobe (AL) glomeruli, course of the output tracts, and their projection in the mushroombody (MB) calyx. A: 3D reconstruction of the major neuropils in the brain of a C. floridanus worker. Central complex, CC; optic lobe, OL; mushroom body, MB. B,C: 3D reconstructions of the antennal-sensory tract specific innervation of glomerular clusters (T1-T7) based on 200 optical sections. B, ventral and $\mathrm{C}$, dorsal view. The color code defines the different sensory tracts (T1-T7) and their innervated glomerular clusters. The T1 cluster (orange) contains $\approx 34$ glomeruli, the T2 cluster (green) $\approx 56$ glomeruli, the T3 cluster (magenta) $\approx 96$ glomeruli, the T4 cluster (light blue) $\approx 78$ glomeruli, the T5 cluster (light green) $\approx 36$ glomeruli, the T6 cluster (dark blue) is the largest cluster with $\approx 128$ glomeruli, and the T7 cluster (yellow) is the smallest cluster with the six largest glomeruli in the AL (see Table 1). D: Ortho slice of a doubly stained AL showing the glomerular innervation of the two output tracts, m- and l-ACT (magenta and green), within the AL Please note that the colors in $\mathrm{D}, \mathrm{E}$ do not reflect connectivity with the glomeruli with similar colors in B. E: Reconstruction of the AL shown in D. Ventral view showing the tract-specific innervation of glomeruli and the position of m- (magenta) and l-ACT (green) PN somata cluster. Glomeruli innervated via the l-ACT (green) are located in the rostral-ventral hemisphere of the AL, m-ACT glomeruli (magenta) innervate the caudal-dorsal hemisphere of the AL. The somata of l-ACT neurons (dark green) form a large cluster at the ventral part of the $\mathrm{AL}$ (ISC1) and a smaller cluster at the rostral rim of the $\mathrm{AL}$ (1SC2; not visible in this view; see Suppl. online material). Somata of the m-ACT neurons (dark magenta) form two clusters, one in the rostralmedial (mSC1) and one in the caudal-lateral region of the AL (mSC2). F: Projection view of an anterograde mass fill of the AL output tracts and their projections in the MB and LH. The m- and l-ACT run to the medial and lateral $\mathrm{MB}$ calyces $(\mathrm{mCa}, \mathrm{lCa})$ and to the $\mathrm{LH}$. Three small ml-ACTs (1-3) project directly to the LH, two of them (ml-ACT 2 and 3) form a "lateral network" (ln, yellow in G) between the MB vertical lobe and the LH. G: 3D-reconstruction of the mass fill shown in F. The l-ACT is shown in green, the m-ACT is in magenta, and all three ml-ACTs in orange. H: Double labeling of the m-ACT (magenta) and l-ACT (green) projections in the medial MB calyx showing a distinct olfactory innervation pattern within the mushroom body calyx lip and collar region. The lip can be separated into three distinct layers according to their innervation (I, II and III). See text for details. Directions: rostral, r; caudal, c; ventral, v; dorsal, d. medial, m; lateral, 1. Scale bars $=100 \mu \mathrm{m}$ in A-G; $50 \mu \mathrm{m}$ in $\mathrm{H}$. 

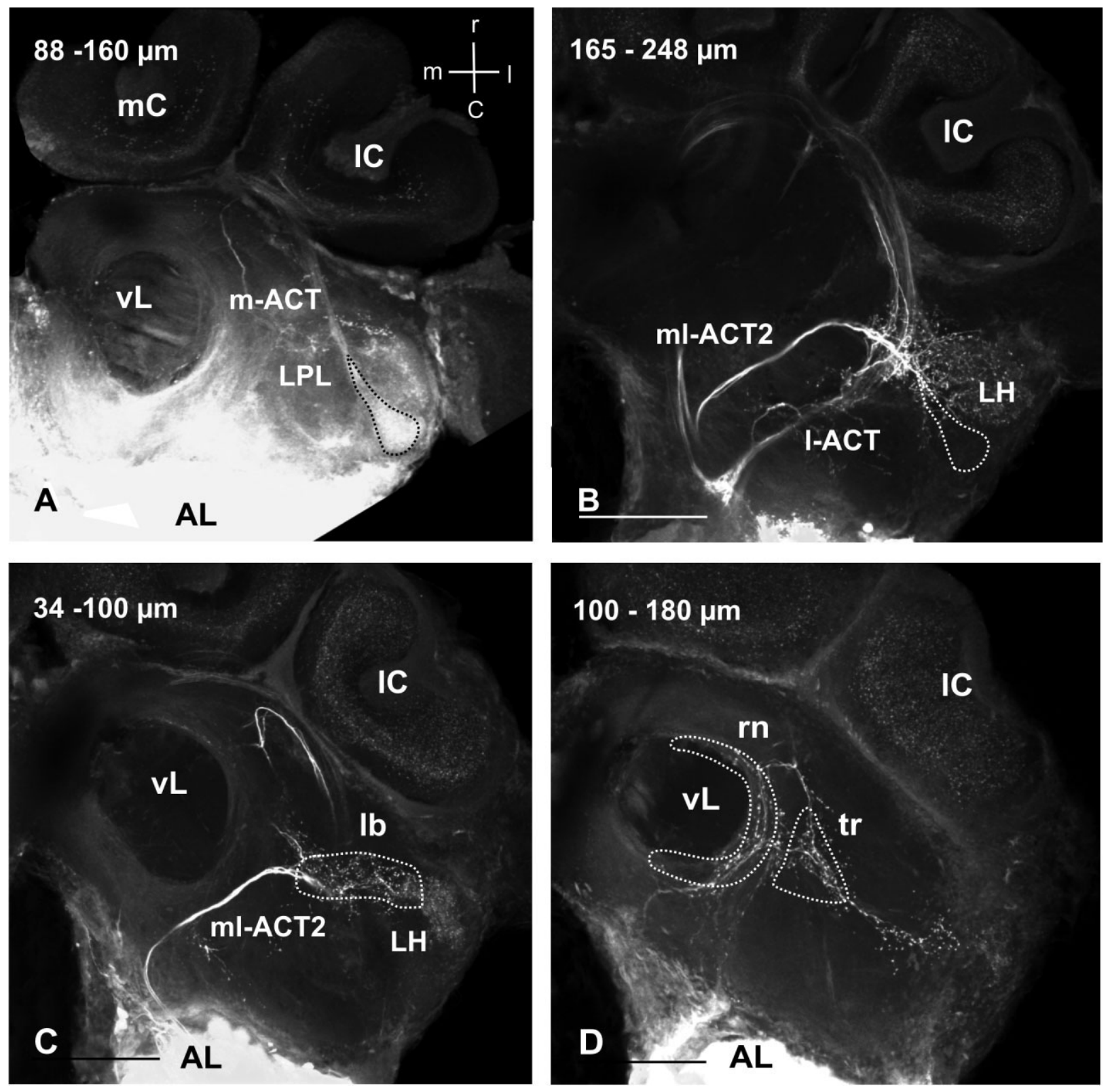

Fig. 3. Spatial organization of ACT projections within the protocerebrum. Projection views at different depths (indicated in A-D) showing the innervation patterns of the ACTs within the lateral horn (LH) and the lateral network within the lateral protocerebral lobe (LPL). A: Club-shaped target region (dashed line) of the m-ACT within the LH. B: Target region of the l-ACT and the ml-ACTs within the LH. The m-ACT region (dashed line) is clearly separated from the

Increasing odor concentrations evoked a higher amplitude of the calcium signal in $\mathrm{ROI}_{\mathrm{cd}+}$ regions and also a longer duration of the calcium signal (Spearman's rank correlation, $\mathrm{R}=0.69, P<0.05$, slope $=0.36$ ). Although increasing odor concentration did not result in a higher ampli-

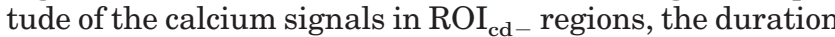
of the calcium signal was also concentration-dependent (Spearman's rank correlation, $\mathrm{R}=0.50, P<0.05$, slope $=$ $0.33)$.

innervation areas of the 1-ACT and ml-ACTs. C,D: Lateral network within the LPL formed by PNs of the ml-ACT 2 and 3. Three different innervation foci can be distinguished: the ring neuropil (rn), triangle (tr), and lateral bridge (lb). Lateral calyx, lC; medial calyx, mC; vertical lobe, vL. Directions: rostral, r; caudal, c; ventral, v; dorsal, d. medial, m; lateral, l. Scale bars $=100 \mu \mathrm{m}$.

\section{DISCUSSION}

This study represents a first comprehensive structurefunction analysis within the central olfactory system of an ant's brain. Our structural data revealed that the AL of $C$. floridanus contains a comparatively high number of olfactory glomeruli (up to 464) supplied via seven distinct antennal sensory tracts. At the output side the AL is subdivided into two almost equally sized hemispheres 
regarding glomeruli innervated by one of two prominent, presumably uniglomerular PN output tracts, the m- and l-ACT. The projections of $\mathrm{m}$ - and l-ACT PNs run in a reverse order to the $\mathrm{MB}$ calyces and the $\mathrm{LH}$, innervating different compartments. In addition, three small tracts (ml-ACT1-3) project through the LPL toward the LH, two of them with side branches in the LPL (ml-ACT2,3) forming a lateral network with several distinct olfactory foci (ring neuropil, triangle, and lateral bridge).

Functional calcium imaging studies of PN activity revealed reproducible glomerular activation patterns in response to pheromonal (alarm, trail) and general odors at about equally high sensitivity. No obvious spatial segregation among pheromonal and nonpheromonal odor activation patterns was observed for the odors tested. Whereas the spatial response patterns to stimulation with trail pheromone (nerolic acid) were remarkably stable over a wide range of intensities $(\approx 8$ log units), the response intensities and especially response durations were dependent of odor intensities.

\section{Antennal lobe design}

The total number of AL glomeruli in C. floridanus is almost 3 -fold higher than in the honeybee (464 vs. 164) and glomeruli in the AL are innervated and organized into seven clusters innervated by seven antennal sensory tracts compared to four main tracts (T1-4) in the honeybee (Abel et al., 2001), or six input tracts when subdivisions of T3 (a-c) are counted as separate tracts as suggested by Kirschner et al. (2006). Despite substantial differences in the antennal sensory input the general innervation pattern of two prominent output tracts (m- and l-ACT) is very similar compared to the situation in the honeybee (Kirschner et al., 2006). The dorsal-rostral part of the AL in C. floridanus is innervated only by l-ACT PNs, the ventral-caudal part exclusively by m-ACT PNs. Compared to the honeybee, both hemispheres are rotated by about $45^{\circ} \mathrm{C}$. The division of the $\mathrm{AL}$ into two hemispheres with each half containing about $50 \%$ of the glomeruli very likely reflects a typical feature of the olfactory pathway in Apocrita (Kirschner et al., 2006). Whether it is a common feature of all hymenoptera needs to be shown in future neuroanatomical studies of other advanced and basal hymenoptera.

Since our calcium imaging data gives only information about the physiological properties of glomeruli belonging to clusters T1-3 on top of the AL, other methods that allow imaging of deeper layers of the AL, such as two photon confocal imaging or electrophysiology studies, are required to gain information about the functional role of the division of the AL into $\mathrm{m}$ - and l-ACT hemispheres. It will be especially interesting to see whether a common picture may emerge from a physiological comparison between the honeybee and the ant. In addition to mapping different categories of odors, the function of a dual pathway could as well be to code different temporal aspects of the stimulus, as suggested by Müller et al. (2002), or to extract different stimulus parameters. To study temporal aspects of odor coding, comparative electrophysiological studies of response profiles are needed.

We did not find any obviously enlarged glomeruli comparable to the macroglomerulus found at the AL entrance in leaf-cutting ants (Kleineidam et al., 2005) or macroglomerular complexes in moths or in honeybee drones (e.g., Hildebrand et al., 1997; Rössler et al., 1999a; Brockmann and Brückner, 2001; Sandoz 2006). This, together with the fact that in our calcium imaging experiments the response patterns to stimulation with pheromonal and nonpheromonal substances were equally sensitive and not obviously segregated indicates that processing of pheromonal and nonpheromonal odors may be combinatorial in the ant $\mathrm{AL}$ (see further discussion of this aspect below).

Does the high number of glomeruli in the $\mathrm{AL}$ of $C$. floridanus indicate more sophisticated olfactory processing compared to, e.g., the honeybee, the fly, or moth? Comparison of the number of odorant receptor (OR) genes in recently sequenced insect genomes of Drosophila melanogaster ( $\approx 62$; Clyne et al., 1999; Robertson et al., 2002; Vosshall et al., 2002), the malaria mosquito Anopheles gambiae $(\approx 79$; Hill et al., 2002$)$, and the honeybee $(\approx 170$; Weinstock et al., 2006) reveal a rough correlation between the number of OR genes and the number of olfactory glomeruli in the AL (43 in Drosphila; Laissue et al., 1999; 61 in Anopheles; Ghaninia et al., 2007; 164 in Apis; Galizia et al., 1999b; Kirschner et al., 2006). Similar relationships were also found in vertebrate olfactory systems (Buck and Axel, 1991). If we assume a similar correlation of OR numbers and AL glomeruli in C. floridanus, we expect a substantially higher number of ORs compared to the honeybee. To further prove this hypothesis would require mapping OR expression to find out whether it may lead to novel glomeruli. The process of glomerulus induction itself, however, is not likely to be directly influenced by ORs because of their late expression in insects (Vosshall et al., 2002). C. floridanus has small optic ganglia compared to relatively large ALs (Fig. 2A). In contrast, the honeybee has elaborated optic ganglia and sophisticated visual capabilities (Gronenberg, 2001; Stach et al., 2004).In this context, evolution of a higher number of glomeruli in highly olfactory ants appears plausible. In the same line, neuroanatomical studies of the more primitive ponerine ant, Harpegnathos saltator, a highly visual predator, revealed a much lower number of $\approx 178$ glomeruli (Hoyer et al., 2005). An interesting hypothesis to follow up in the future is that the evolution of new OR genes may come along with the formation of new sensory tracts and clusters of glomeruli in the AL and/or the increase/decrease in the size of existing ones. Future comparison of more closely related ant species of the subfamily Formicinae and future genomic data may reveal further insight into the question.

Structural comparison of sensory-tract-specific glomerular clusters in C. floridanus indicates some similarities (possibly homologies), but also clear differences compared to the situation in the honeybee (Kirschner et al., 2006). The T7 cluster of C. floridanus shares striking similarities with the T4 cluster in the honeybee: both possess six large glomeruli at the dorsal end of the AL with a unique sensory innervation pattern and with one or two glomeruli neither connected to the m-ACT nor to the l-ACT. If the two clusters turn out to be homologous, the perseverance of their anatomy throughout the Apocrita lineage would predict an important functional significance of these six glomeruli. Physiological, neurochemical, and molecular characterization of this deep cluster, therefore, will be very elucidating.

The T3 cluster of C. floridanus shares similarities with the T3a cluster after Kirschner et al. (2006), especially its position at the ventral-medial AL and the division of its glomeruli to both ACT-hemispheres. The best candidates 

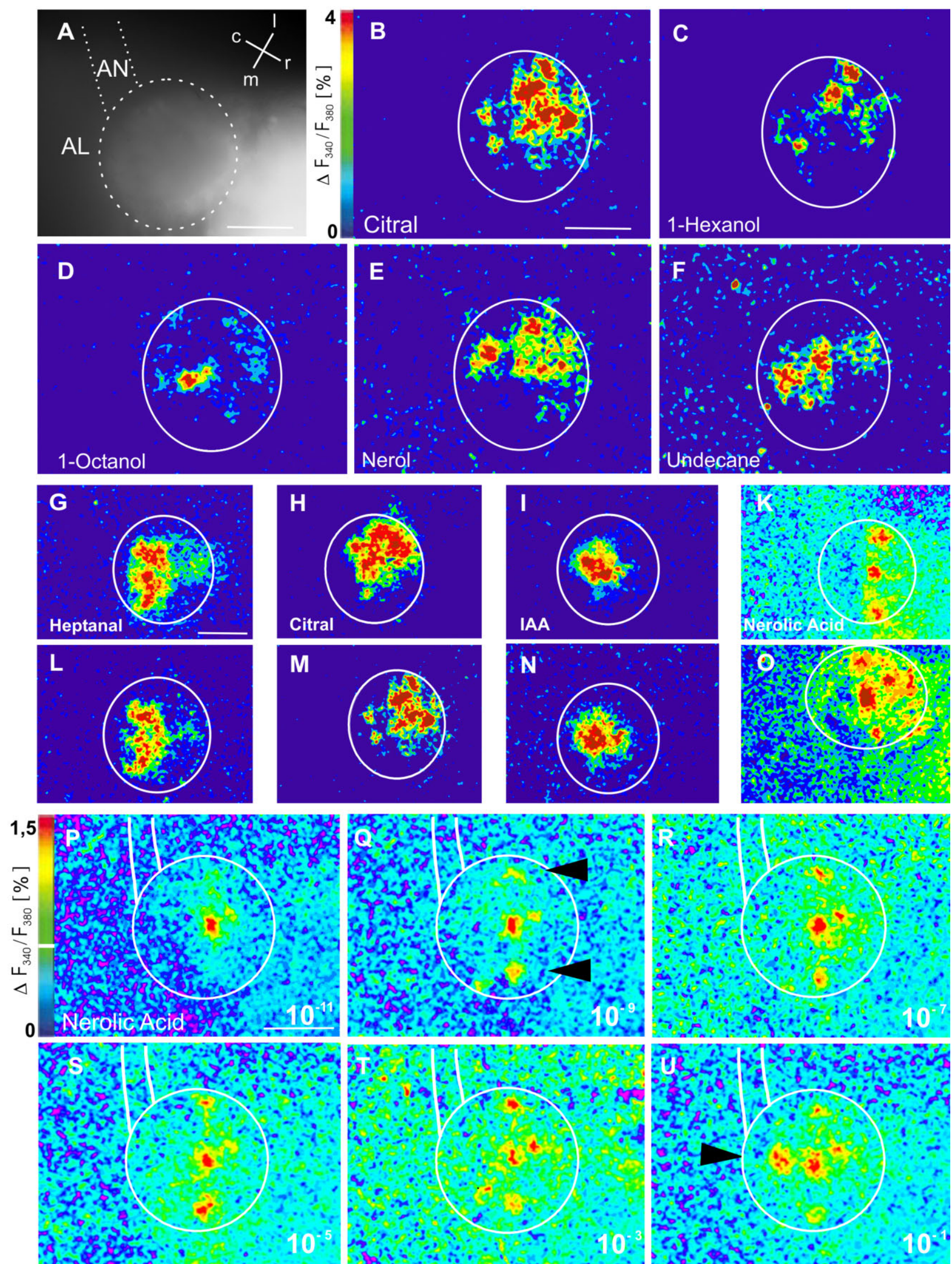
for being homologous to the honeybee $\mathrm{T} 1$ cluster are $\mathrm{T} 1,2$, and 4 in C. floridanus: all three together make up the rostral-lateral part of the $\mathrm{AL}$, like in the honeybee if one considers the $45^{\circ}$ shift of the ant $\mathrm{AL}$, and they all belong to the l-ACT hemisphere. On the opposite side of the AL, the large T6 cluster in C. floridanus shares five anatomical features with the very small T3b cluster in the honeybee (after Kirschner et al., 2006): first a position at the medialcaudal AL flanking the AN, second a sensory tract that approaches the glomeruli from the periphery of the $\mathrm{AL}$, third a clear separation from the other glomeruli clusters, fourth a composition of relatively small glomeruli, and fifth the affiliation to the m-ACT hemisphere. The most striking difference of the T6 cluster in C. floridanus compared to T3b cluster in the honeybee is the high number of $\approx 128$ glomeruli indicating that this could be a spot of strong evolutionary proliferation of glomeruli in the ant AL.

\section{Odor representation in the AL}

Calcium imaging in the AL revealed odor-induced activity of PNs. We employed the retrograde loading technique, originally developed in the honeybee by Sachse and Galizia (2002), for the first time successfully in the ant. Although both l- and m-ACT PNs were backfilled with our technique, our neuroanatomical analyses show that the field of view in calcium imaging experiments was restricted to the ventral part of the AL almost exclusively innervated by l-ACT PNs (with only a few exceptions of m-ACT glomeruli in the T3 cluster; see Fig. 2E). Therefore, all further conclusions are mostly limited to l-ACT PNs.

Odor-specific activation patterns in primary olfactory centers were described for a number of invertebrate and vertebrate species (e.g., Rodrigues, 1988; Friedrich and Korsching, 1997; Joerges et al., 1997; Rubin and Katz, 1999; Hansson et al., 2003; Carlsson et al., 2005), but only one earlier study in a closely related Camponotus species (Galizia et al., 1999a). Activation patterns in response to different odors were overlapping, indicating that some glomeruli contribute to the activation pattern elicited by

Fig. 4. Projection-neuron calcium responses in the antennal lobe. A: Fura-2 raw fluorescence image of the antennal lobe (AL). The position of the antennal nerve $(\mathrm{AN})$ and the spatial directions are indicated. The spatial directions are valid for all false-color coded images. B-F: False-color coded images showing activity areas in the $\mathrm{AL}$ of $C$. floridanus in response to different odors. The calcium signals were recorded from PNs of the AL loaded with Fura-2 dextran. $500 \mathrm{nl}$ (dilution $10^{-1}$ ) for all odors. The different odors elicit odor-specific activity areas within one ant. G-O: The same odors elicit similar activity areas in different ants. Examples showing activity areas in response to heptanal (dilution of $10^{-1}$ ), isoamylacetate (IAA; dilution of $10^{-1}$ ), citral (dilution of $10^{-1}$ ), nerolic acid (dilution of $10^{-9}$ ) in two different ants. P-U: Response patterns to the trail pheromone component nerolic acid presented at different concentrations. High concentration leads to the recruitment of additional activation spots (arrows in Q and U). The dilutions are given in the images. Note that the activity areas in response to general odors covered large parts of the AL (B,H,M, citral; E, nerol; G,L, heptanal) or were more confined with only few activity spots (C, 1-hexanol; D, 1-octanol). Pheromones ( $\mathrm{F}$, the alarm pheromone $\mathrm{n}$-undecane; $\mathrm{K}, \mathrm{O}, \mathrm{P}-\mathrm{U}$, the trail pheromone component nerolic acid) elicited qualitative similar activity areas compared to general odors. False-colors are scaled in images B-F as shown in B, images G-O as shown in G, and images in $\mathrm{P}-\mathrm{U}$ as shown in P. caudal, c; lateral, l; medial, m; rostral, r. Scale bars $=100 \mu \mathrm{m}$. different odors. We further were able to show that odorspecific activity patterns are conserved between individuals, similar to findings in the honeybee (Galizia et al., 1999b).

Only in some cases could calcium signals from the PNs be assigned to individual glomeruli (activity spots). Large activity regions indicate that the odor used for stimulation was represented in several adjacent glomeruli rather than in isolated single glomeruli of the AL. Studies in the honeybee indicate that adjacent glomeruli may have similar response profiles (Sachse et al., 1999). In our study the size of cohesive activity regions covering several glomeruli remained constant over a large range of concentrations, and in most cases was only marginally smaller at odor concentration threshold. Are the large activity regions the result of scattered fluorescence from activated glomeruli out of focus, e.g., in deeper layers of the AL? Glomeruli in the $\mathrm{AL}$ of $C$. floridanus are clustered (Figs. 1, 2), and some glomeruli may be above or below the focal plane during calcium imaging. Repeated stimulation with the same odor at different focal planes revealed large activity regions also in z-direction, and no confined activity spots were found up to a depth of $80 \mu \mathrm{m}$ (data not shown). This indicates a large or even complete overlap of response profiles in at least some neighboring glomeruli. Other glomeruli seemed to have a more distinct response profile compared to their adjacent glomeruli. For example, with an increasing concentration of nerolic acid, some activity spots appeared at low concentration and remained constant in size up to high odor concentrations. In this case, no glomeruli next to the activity spots were recruited to the activation pattern. In our study we used only singlecomponent odors, and it remains to be shown whether odor mixtures may elicit a more distinct activation pattern across glomeruli within the same activity region by processing of odor information in the AL network.

The activity patterns in response to (nonsex) pheromones (or pheromone components) were distributed across the field of view, and there was no obvious qualitative difference compared to the activation patterns in response to general odors. Even very low pheromone concentrations elicited a distributed activation pattern in the $\mathrm{AL}$ and no distinct cluster of pheromone specific glomeruli was found. Yamagata et al. (2006) using electrophysiological recording and staining of $\mathrm{AL}$ neurons described "alarm pheromone-sensitive" glomeruli in another Camponotus species that form a cluster in the dorsal most part of the AL. This particular glomerular cluster was not accessible in our imaging experiments. Nevertheless, in contrast to this our calcium imaging experiments revealed a rather distributed representation of n-undecane in $C$. floridanus, and we conclude that for n-undecane as well as for nerolic acid pheromone-specific clusters of glomeruli do not exist in the AL of workers. This confirms and extends similar observations from a calcium imaging study in Camponotus rufipes (Galizia et al., 1999a), and is consistent with the rather distributed representation of nonsex pheromones in glomeruli within the $\mathrm{AL}$ of the honeybee (Joerges et al., 1997; Galizia et al., 1999c). It remains to be shown whether this arrangement is advantageous or even necessary to allow context-specific behavioral responses to pheromones as described for ants (Hölldobler and Wilson, 1990; Knaden and Wehner, 2003). Differential neuromodulatory innervation of the AL (Dacks et al., 2006; Ziegler et al., 2007) are likely to promote primary process- 

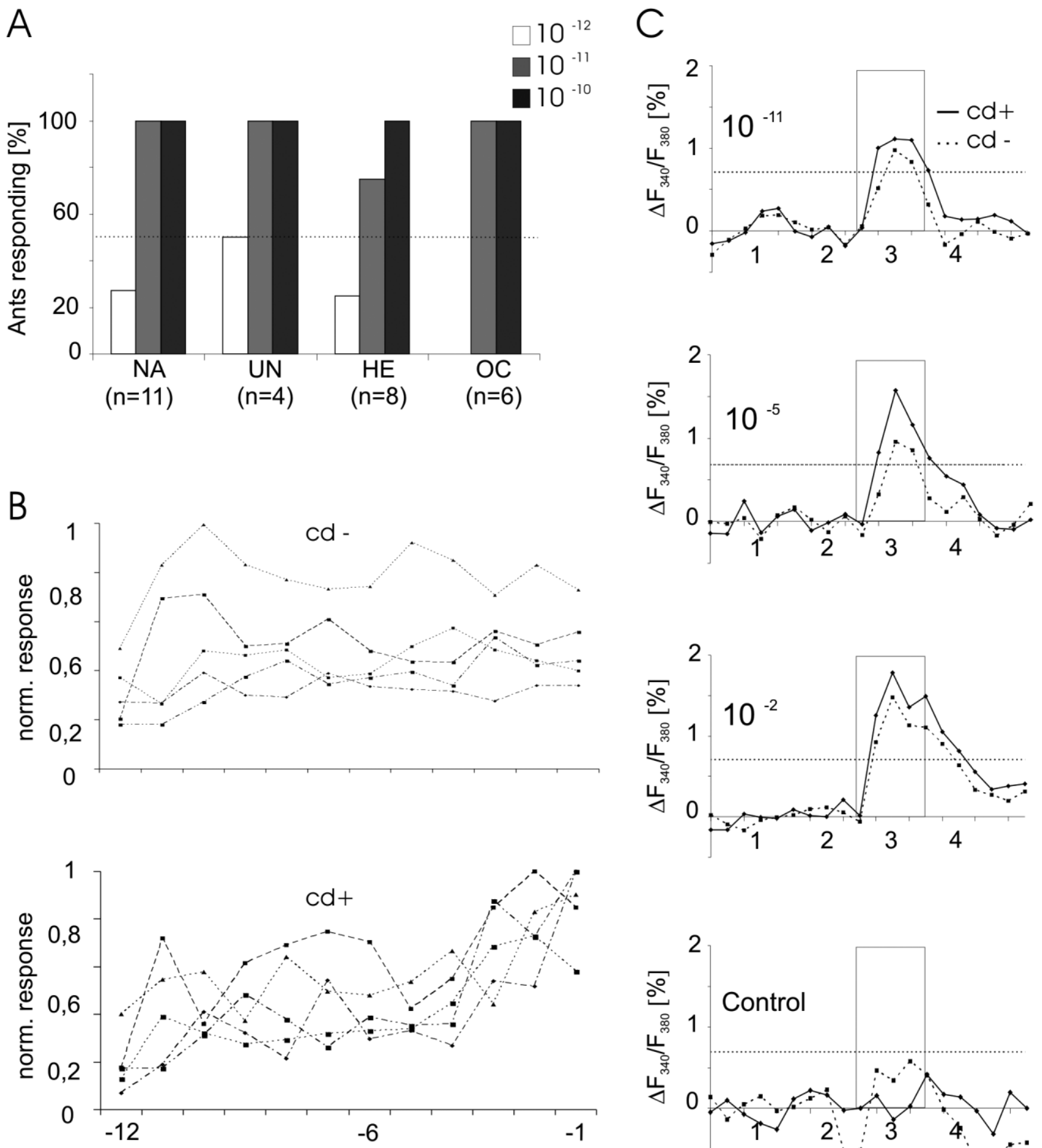

\section{log dilution}

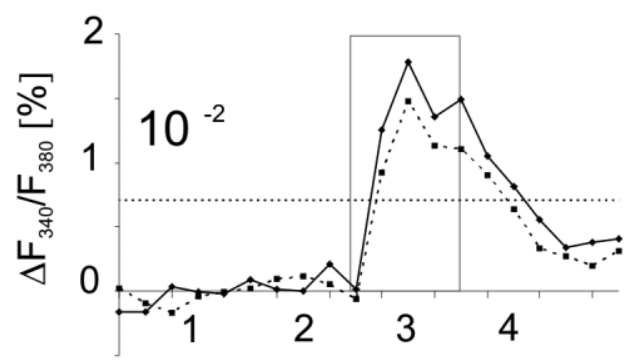

Fig. 5. Sensitivity of odor responses and intensity dependence of responses to nerolic acid. A: Response-threshold odor concentration for pheromones (NA, nerolic acid; UN, n-undecane) and general odors (HE, 1-hexanol; OC, 1-octanol). Percentage of animals in which a calcium signal (activity areas) could be measured in response to different odor concentrations (dilutions: white bars, $10^{-12}$; gray bars, $10^{-11}$; black bars $10^{-10}$ ). Loading the odor cartridge with $10^{-11}$ dilution of any of the four odors was sufficient to elicit a calcium response in at least $80 \%$ of the animals (n, number of animals tested). B: Normalized amplitudes (see Materials and Methods) of the calcium re-

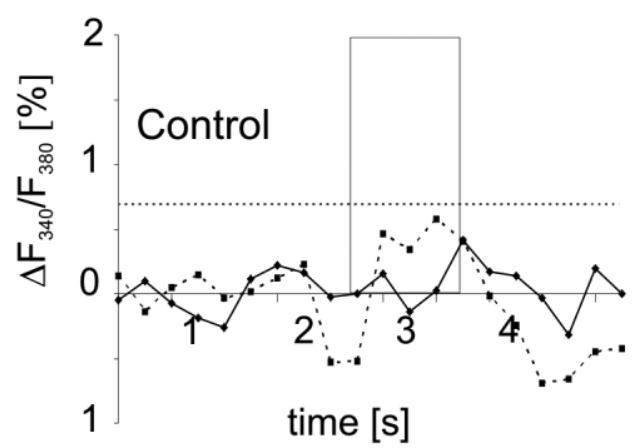

sponses to nerolic acid in five concentration-dependent $(\mathrm{cd}+)$ and five concentration-independent (cd-) ROIs within the antennal lobe of five animals across a range of odor concentrations (dilution: $10^{-12}$ to $\left.10^{-1}\right)$. C: Temporal dynamics of the calcium responses to nerolic acid in one concentration-dependent $(\mathrm{cd}+)$ and one concentrationindependent $(\mathrm{cd}-)$ ROI within the antennal lobe of one animal at three different odor concentrations $\left(10^{-11}, 10^{-5}\right.$, and $\left.10^{-2}\right)$ and the control stimulus (solvent only). Threshold level of $40 \%$ is indicated by the horizontal dotted line. The response duration was longer at higher odor concentrations compared to lower concentrations. 
ing of pheromone information and certainly need further investigation.

The concentration thresholds we measured were at extremely low concentrations. Odor loading of the filter paper in the range of $0.05 \mathrm{pg}$ (equivalent to dilutions of $10^{-11}$ ) was sufficient to elicit reliable calcium amplitudes in $\mathrm{PNs}$, and concentration thresholds were similar for both pheromones and general odors. Although it is difficult to compare data from different laboratories using different types of odor stimulation devices (e.g., variation in air flow velocity and volume), the odor sensitivity in ants seems to be 5 log units higher compared to what was described for the honeybee (Sachse and Galizia, 2003). In addition, in the honeybee the spatial activation pattern was shown to be concentration invariant only over a limited range of concentrations. Our results in C. floridanus indicate that the spatial activation pattern in response to the trail pheromone component is largely invariant over a rather large concentration range of $\approx 8 \mathrm{log}$ units. Only at the lowest and highest concentrations, glomeruli disappeared or additional ones were recruited. Concentrationdependent activity patterns were also described in other species (Rubin and Katz, 1999; Johnson and Leon, 2000; Meister and Bonhoeffer, 2001, Fu $\beta$ and Korsching, 2001; Wachowiak and Cohen, 2001). The main reason for the change in activity patterns at the level of the AL or olfactory bulb probably is that the receptive range of individual ORNs is concentration-dependent and broadens with increasing odor concentrations (Firestein et al., 1993; Duchamp-Viret et al., 2000; de Bruyne et al., 2001). The perceived odor quality can depend on odor concentration, a phenomenon known in human psychophysics (GrossIsserhoff et al., 1988) and from experiments in Drosophila (Rodrigues and Siddiqi, 1983; Stensmyr et al., 2003). In ants, concentration-dependent responses may result, e.g., in a well-defined behavioral sequence when approaching a (alarm) pheromone source (active space hypothesis; Bradshaw and Howse, 1984; Hölldobler and Wilson, 1990). The idea that indeed the activity pattern found in the AL corresponds to perceptual measures recently received strong support from a study in the honeybee (Guerrieri et al., 2005). Interestingly, in response to trail pheromone we found activity spots only adding to the pattern at very high or low concentrations. Sampling of a chemical trail with antennation movements may cause large fluctuations at ORNs; therefore, it may be important to maintain a constant spatial pattern at the level of the PN output in order to stabilize odor quality perception. Higher concentrations of the trail pheromone, on the other hand, enhance the probability of a worker to follow a trail. Therefore, both the maintenance of a constant quality perception and, at the same time, assessing odor intensity is important for trail-following behavior. Our results indicate that the response duration may be a reliable measure for coding odor intensity. Concentration-invariant activation patterns together with graded calcium-signal amplitudes, as also described in the honeybee, led to the assumption that the activity pattern is the important parameter for assessing odor quality while the peak activity of the PNs relates to odor intensity (Galizia and Menzel, 2001). For some activity regions, but not for all, we found graded calcium-signal amplitudes with increasing odor concentrations. In other cases, activity regions with almost constant calcium-signal amplitudes over a wide range of concentrations had considerably stronger ampli- tudes at the highest concentration than at any other concentration (Fig. 5B). In case of high basal intracellular calcium concentration, weak neural responses may already lead to a saturation of the Fura-2 signal. In these cases the calcium amplitude would not increase with increasing response strength, but the duration would. However, the fact that even in cd-cases we observed a slight increase in the amplitude only at the highest concentration indicates that the Fura-2 signal was not saturated. In contrast to variations with respect to graded amplitudes, we found graded response durations in all cases. This indicates that response duration might be an important parameter for assessing odor concentration, although one has to keep in mind that the duration of the Fura-2 signal may be strongly influenced by both the strength (spike rate) and the duration of a neural response. Further electrophysiological analyses at the single neuron level are required to fully clarify this aspect in odor coding.

Odor information is relayed to higher brain regions via a combinatorial code of PN activation. Although it is not fully understood how odor quality and intensity are coded by the population of $\mathrm{PNs}$, it seems plausible that the temporal structure of PN activity is important. Mixtures of odors lead to a synchronization of pheromone-sensitive PNs in moths (Lei et al., 2002), and in the locust oscillatory synchronization of activity is considered as an important feature for odor coding and recognition (Laurent, 2002). Prolonged activation of PNs in response to higher odor concentrations might enhance temporally coordinated PN activity, and temporal coordination may be important for both odor quality and intensity coding (Lei et al., 2004). In this respect, spatial separation of $\mathrm{m}$ - and l-ACT PN pathways may be an important addition in the Apocritan lineage reflecting the evolution of advanced processing of a large variety of both chemical communication signals and environmental odors.

\section{ACKNOWLEDGMENTS}

We thank Wulfila Gronenberg for help with neuroanatomical tracing techniques, Malu Obermayer for expert technical assistance, Mona Alzheimer for 3Dreconstruction assistance, and Annett Endler for help with rearing of Camponotus floridanus.

\section{LITERATURE CITED}

Abel R, Rybak J, Menzel R. 2001. Structure and response patterns of olfactory interneurons in the honeybee, Apis mellifera. J Comp Neurol 437:363-383

Bradshaw JWS, Howse PE. 1984. Sociochemicals of ants. In: Bell WJ, Carde RT, editors. Chemical ecology of insects. London: Chapman \& Hall. p 429-473.

Brockmann A, Brückner D. 2001. Structural differences in the drone olfactory system of two phylogenetically distant Apis species, A. florea and $A$. mellifera. Naturwissenschaften 88:78-81.

Buck L, Axel R. 1991. A novel multigene family may encode odorant receptors: a molecular basis for odor recognition. Cell 65:175-187.

Carlsson MA, Hansson BS. 2003. Dose-response characteristics of glomerular activity in the moth antennal lobe. Chem Senses 28:269-278.

Carlsson MA, Galizia CG, Hansson BS. 2002. Spatial representation of odours in the antennal lobe of the moth Spodoptera littoralis (Lepidoptera: Noctuidae). Chem Senses 27:231-244.

Carlsson MA, Knusel P, Verschure PFMJ, Hansson BS. 2005. Spatiotemporal $\mathrm{Ca} 2+$ dynamics of moth olfactory projection neurones. Eur J Neurosci 22:647-657.

Christensen T, Hildebrand J. 1987. Male-specific, sex-pheromone-selective 
projection neurons in the antennal lobes of the moth Manduca sexta. J Comp Physiol A 160:553-569.

Clyne P, Certel S, de Bruyne M, Zaslavsky L, Johnson W, Carlson J. 1999 The odor specificities of a subset of olfactory receptor neurons are governed by Acj6, a POU-domain transcription factor. Neuron 22:339347.

Dacks AM, Christensen TA, Hildebrand JG. 2006. Phylogeny of a serotonin-immunoreactive neuron in the primary olfactory center of the insect brain. J Comp Neurol 498:727-746.

de Bruyne M, Foster K, Carlson JR. 2001. Odor coding in the Drosophila antenna. Neuron 30:537-552.

Duchamp-Viret P, Duchamp A, Chaput MA. 2000. Peripheral coding in the rat and frog: quality and intensity specification. J Neurosci 20:23832390 .

Firestein S. 1993. Olfactory receptor neuron signaling. ACS Sym Ser 825:110-122.

Friedrich RW, Korsching SI. 1997. Combinatorial and chemotopic odorant coding in the zebrafish olfactory bulb visualized by optical imaging. Neuron 18:737-752.

$\mathrm{Fu} \beta$ SH, Korsching SI. 2001. Odorant feature detection: Activity mapping of structure response relationship in the zebrafish and olfactory bulb. J Neurosci 21:8396-8401.

Galizia CG, Menzel R. 2001. The role of glomeruli in the neural representation of odors: results from optical recording studies. J Insect Physiol 47:115-130.

Galizia CG, Menzel R, Hölldobler B. 1999a. Optical imaging of odor-evoked glomerular activity patterns in the antennal lobes of the ant Camponotus rufipes. Naturwissenschaften 86:533-537.

Galizia CG, Sachse S, Rappert A, Menzel R. 1999b. The glomerular code for odor representation is species specific in the honeybee Apis mellifera. Nat Neurosci 2:473-478.

Galizia GC, McIlwrath SL, Menzel R. 1999c. A digital three-dimensional atlas of the honeybee antennal lobe based on optical sections acquired by confocal microscopy. Cell Tissue Res 295:383-394.

Ghaninia M, Hansson BS; Ignell R. 2007. The antennal lobe of the African malarian mosquito, Anopheles gambiae-innervation and threedimensional reconstruction. Arthropod Struct Dev 36:29-39.

Gronenberg W. 1999. Modality specific segregation of input to ant mushroom bodies. Brain Behav Evol 54:85-95.

Gronenberg W. 2001. Subdivisions of hymenopteran mushroom body calyces by their afferent supply. J Comp Neurol 435:474-489.

Gross-Isseroff R, Lancet D. 1988. Concentration-dependent changes of perceived odor quality. Chem Senses 13:191-204.

Guerrieri F, Schubert M, Sandoz JC, Giurfa M. 2005. Perceptual and neural olfactory similarity in honeybees. PLOS Biol 3:718-732.

Haak U, Hölldobler B, Bestmann HJ, Kern F. 1996. Species-specificity in trail pheromones and dufour's gland contents of Camponotus atriceps and C. floridanus (Hymenoptera: Formicidae). Chemoecology 7:85-93.

Hansson BS, Anton S. 2000. Function and morphology of the antennal lobe: new developments. Annu Rev Entomol 45:203-231.

Hansson BS, Christensen TA, Hildebrand JG. 1991. Functionally distinct subdivisions of the macroglomerular complex in the antennal lobe of the male sphinx moth Manduca sexta. J Comp Neurol 312:264-278.

Hansson BS, Ljundberg H, Hallberg E, Lofstedt C. 1992. Functional specialization ot olfactory glomeruli in a moth. Science 256:1313-1315.

Hansson BS, Carlsson MS, Kalinova B. 2003. Olfactory activation patterns in the antennal lobe of the sphinx moth, Manduca sexta. J Comp Physiol A 189:301-308.

Hildebrand JG, Shepherd GM. 1997. Mechanisms of olfactory discrimination: converging evidence for common principles across phyla. Annu Rev Neurosci 20:595-631.

Hill CA, Fox AN, Pitts RJ, Kent LB, Tan PL, Chrystal MA, Cravchik A, Collins FH, Robertson HM, Zwiebel LJ. 2002. G-protein-coupled receptors in Anopheles gambiae. Science 298:176-178.

Hölldobler B. 1995. The chemistry of social regulation: multicomponent signals in ant societies. Proc Natl Acad Sci U S A 92:19-22.

Hölldobler B, Wilson EO. 1990. The ants. Cambridge, MA: Harvard University Press.

Hoyer SC, Liebig J, Rössler W. 2005. Biogenic amines in the ponerine ant Harpegnathos saltator: serotonin and dopamine immunoreactivity in the brain. Arthropod Struct Dev 34:429-440.

Joerges J, Küttner A, Galizia GC, Menzel R. 1997. Representation of odours and odour mixtures visualized in the honeybee brain. Nature $387: 285-288$
Johnson BA, Leon M. 2000. Modular representations of odorants in the glomerular layer of the rat olfactory bulb and the effects of stimulus concentration. J Comp Neurol 422:496-509.

Kirschner S, Kleineidam CJ, Zube C, Rybak J, Grünewald B, Rössler W 2006. Dual olfactory pathway in the honeybee, Apis mellifera. J Comp Neurol 499:933-952

Kleineidam CJ, Obermayer M, Halbich W, Rössler W. 2005. A macroglomerulus in the antennal lobe of leaf-cutting ant workers and its possible functional significance. Chem Senses 30:383-392.

Knaden M, Wehner R. 2003. Nest defense and conspecific enemy recognition in the desert ant Cataglyphis fortis. J Insect Behav 6:717-730.

Lahav S, Soroker V, Hefetz A, Vander Meer RK. 1999. Direct behavioral evidence for hydrocarbons as ant recognition discriminators. Naturwissenschaften 86:246-249.

Laissue PP, Reiter C, Hiesinger PR, Halter S, Fischbach KS, Stocker RF. 1999. Three-dimensional reconstruction of the antennal lobe in Drosophila melanogaster. J Comp Neurol 405:543-552.

Laurent G. 2002. Olfactory network dynamics and the coding of multdimensional signals. Nat Rev Neurosci 3:884-895.

Lei H, Christensen TA, Hildebrand JG. 2002. Local inhibition modulates odor-evoked synchronization of glomerulus-specific output neurons. Nat Neurosci 5:557-565.

Lei H, Christensen TA, Hildebrand JG. 2004. Spatial and temporal organization of ensemble representations for different odor classes in the moth antennal lobe. J Neurosci 24:11108-11119.

Lenoir A, Fresneau D, Errard C, Hefetz A. 1999. Individuality and colonial identity in ants: the emergence of the social representation concept. In Detrain C, Deneubourg JL, Pasteels JM, editors. Information processing in social insects. Berlin: Birkhäuser. p 219-237.

Malun D, Waldow U, Kraus D, Boeckh J. 1993. Connections between the deutocerebrum and the protocerebrum, and neuroanatomy of several classes of deutocerebral projection neurons in the brain of male Periplaneta americana. J Comp Neurol 329:143-162.

Meister M, Bonhoeffer T. 2001. Tuning and topography in an odor map on the rat olfactory bulb. J Neurosci 21:1352-1360.

Mombaerts P, Wang F, Dulac C, Chao SK, Nemes A, Mendelsohn M Edmonson J, Axel R. 1996. Visualizing an olfactory sensory map. Cell $87: 675-686$

Müller D, Abel R, Brandt R, Zockler M, Menzel R. 2002. Differentia parallel processing of olfactory information in the honeybee, Apis mellifera L. J Comp Physiol A 188:359-370.

Peele P, Ditzen M, Menzel R, Galizia CG. 2006. Appetitive odor learning does not change olfactory coding in a subpopulation of honeybee antennal lobe neurons. J Comp Physiol A 192:1083-1103.

Robertson HM, Warr CG, Carlson JR. 2003. Molecular evolution of the insect chemoreceptor gene superfamily in Drosophila melanogaster. Proc Natl Acad Sci U S A 100:14537-14542.

Rodrigues V. 1988. Spatial coding of olfactory information in the antennal lobe of Drosophila melanogaster. Brain Res 453:299-307.

Rodrigues V, Siddiqi O. 1978. Genetic analysis of chemosensory pathway. Proc Ind Acad Sci 87B:147-160.

Rospars JP, Hildebrand JG. 2000. Sexually dimorphic and isomorphic glomeruli in the antennal lobes of the sphinx moth Manduca sexta. Chem Senses 25:119-129.

Rössler W, Randolph PW, Tolbert LP, Hildebrand JG. 1999a. Axons of olfactory receptor cells of transsexually grafted antennae induce development of sexually dimorphic glomeruli in Manduca sexta. J Neurobiol 38:521-541.

Rössler W, Oland LA, Higgins MR, Hildebrand JG, Tolbert LP. 1999b. Development of a glia-rich axon-sorting zone in the olfactory pathway of the moth Manduca sexta. J Neurosci 19:9865-9877.

Rubin BD, Katz LC. 1999. Optical imaging of odorant representations in the mammalian olfactory bulb. Neuron 23:499-511.

Sachse S, Galizia CG. 2003. The coding of odour-intensity in the honeybee antennal lobe: local computation optimizes odour representation. Eur J Neurosci 18:2119-2132.

Sachse S, Rappert A, Galizia CG. 1999. The spatial representation of chemical structures in the AL of honeybees: steps towards the olfactory code. Eur J Neurosci 11:3970-3982.

Sandoz JC. 2006. Odour-evoked responses to queen pheromone components and to plant odours using optical imaging in the antennal lobe of the honey bee drone Apis mellifera L. J Exp Biol 209:3587-3598.

Sandoz JC, Galizia CG, Menzel R. 2003. Side-specific olfactory conditioning 
leads to more specific odor representation between sides but not within sides in the honeybee antennal lobes. Neuroscience 120:1137-1148.

Singer TL. 1998. Roles of hydrocarbons in the recognition systems of insects. Am Zool 38:394-405.

Stach S, Bernard J, Giurfa M. 2004. Local-feature assembling in visual pattern recognition and generalization in honeybees. Nature 429:758761.

Stensmyr MC, Giordano E, Balloi A, Angioy AM, Hansson BS. 2003. Novel natural ligands fro Drosophila olfactory receptor neurons. J Exp Biol 206:715-724.

Strausfeld NJ. 2002. Organization of the honey bee mushroom body: Representation of the calyx within the vertical and gamma lobes. J Comp Neurol 450:4-33.

Vassar R, Chao SK, Sitcheran R, Nunez JM, Vosshall LB, Axel R. 1994. Topographic organization of sensory projections to the olfactory bulb. Cell 79:981-991.

Vickers NJ, Christensen TA, Hildebrand JG. 1998. Combinatorial odor discrimination in the brain: attractive and antagonist odor blends are represented in distinct combinations of uniquely identifiable glomeruli. J Comp Neurol 400:35-56.
Vosshall LB. 2002. How the brain sees smells. Dev Cell 1:588-590.

Vosshall LB, Wong AM, Axel R. 2000. An olfactory sensory map in the fly brain. Cell 102:147-159.

Wachowiak M, Cohen LB. 2001. Representation of odorants by receptor neuron input to the mouse olfactory bulb. Neuron 32:723-735.

Wang JW, Wong AM, Flores J, Vosshall LB, Axel R. 2003. Two-photon calcium imaging reveals an odor-evoked map of activity in the fly brain. Cell 112:271-282.

Weinstock GM, Robinson GE, Gibbs RA et al. 2006. Insights into social insects from the genome of the honeybee Apis mellifera. Nature 443: 931-949.

$\mathrm{Xu}$ FQ, Greer CA, Shepherd GM. 2000. Odor maps in the olfactory bulb. J Comp Neurol 422:489-495.

Yamagata N, Nishino H, Mizunami M. 2006. Pheromone-sensitive glomeruli in the primary olfactory centre of ants. Proc Biol Sci 273:2219-2225.

Ziegler C, Starke NK, Zube C, Kirschner S, Rössler W. 2007. Neuromodulation and synaptic plasticity within olfactory centers in the brain of the carpenter ant, Camponotus floridanus. Proc 31st Göttingen Neurobiology Conference; p 131. 